\title{
Sensitivity to Physical and Numerical Aspects of Large-Eddy Simulation of Stratocumulus
}

\author{
GEORGIOS MATHEOU \\ Department of Mechanical Engineering, University of Connecticut, Storrs, Connecticut \\ JOÃO TEIXEIRA \\ Jet Propulsion Laboratory, California Institute of Technology, Pasadena, California
}

(Manuscript received 16 August 2018, in final form 26 March 2019)

\begin{abstract}
A series of numerical experiments where both physical and numerical model parameters are varied with respect to a reference setup is used to investigate the physics of a stratocumulus cloud and the performance of a large-eddy simulation (LES) model. The simulations show a delicate balance of physical processes with some sensitivities amplified by numerical model features. A strong feedback between cloud liquid, cloud-top radiative cooling, and turbulence leads to slow grid convergence of the turbulent fluxes. For a methodology that diagnoses cloud liquid from conserved variables, small errors in the total water amount result in large liquid water errors, which are amplified by the cloud-top radiative cooling leading to large variations of buoyancy forcing. In contrast, when the liquid-radiation-buoyancy feedback is not present in simulations without radiation, the turbulence structure of the boundary layer remains essentially identical for grid resolutions between 20 and $1.25 \mathrm{~m}$. The present runs suggest that the buoyancy reversal instability significantly enhances the entrainment rate. Even though cloud-top radiative cooling is regarded as a key attribute of stratocumulus, the present simulations suggest that surface fluxes and surface shear significantly contribute to the total turbulent kinetic energy. Turbulence spectra exhibit inertial range scaling away from the confinement effects of the surface and inversion. Fine grid resolution LESs agree with observations, especially with respect to cloud liquid and vertical velocity variance, and exhibit grid convergence without any model tuning or ad hoc model choices.
\end{abstract}

\section{Introduction}

Stratocumulus clouds $(\mathrm{Sc})$ have a large impact on Earth's radiative balance because they cover about onequarter of Earth's surface and strongly reflect incoming solar radiation while having a small effect on outgoing longwave radiation (e.g., Hartmann et al. 1992; Bretherton 1997; Stevens 2005; Wood 2012). Sc is one of the most studied cloud systems (Stevens 2005) with several observational campaigns (e.g., Lenschow et al. 1988; Stevens et al. 2003a; Malinowski et al. 2013) and modeling studies (e.g., Moeng et al. 1992, 1996; Moeng 2000; Stevens et al. 2000, 2005; Yamaguchi and Randall 2008; Chung et al. 2012; Yamaguchi et al. 2013; Van der Dussen et al. 2014; de Lozar and Mellado 2015; Mellado et al. 2018) contributing to our knowledge of the boundary layer physics. However, the parameterization of the

Corresponding author: Georgios Matheou,matheou@uconn.edu stratocumulus-topped boundary layer in global circulation models remains challenging (e.g., Bony and Dufresne 2005; Bretherton 2015; Tsushima et al. 2016), mainly because of limitations in the physical understanding of the factors controlling Sc cloudiness and entrainment.

The growth of computing power combined with advances in modeling methods is yielding high-fidelity simulations of the atmospheric boundary layer with large-eddy simulation (LES) often used as the currently best available modeling methodology. Typically, LES is sufficiently reliable and it is used to gain insight into boundary layer physics and to inform the development and evaluation of coarse-grained models. However, LES of Sc has been more challenging compared to other boundary layer types, such as cumulus-topped boundary layers (e.g., Siebesma et al. 2003; Stevens et al. 2005; Blossey et al. 2013), with Sc cloud cover, liquid water path, and entrainment exhibiting large variations between models. 
Even though the challenges in accurately simulating Sc are pervasive, the causes are not clear. A main goal of the present study is to understand the source of difficulty in LES of Sc and investigate model performance. Previous studies have hypothesized that a primary cause is the sharp (i.e., large temperature jump in a thin layer) inversion (e.g., Bretherton 2015; Pressel et al. 2017). The boundary layer-free troposphere interface at the cloud-top inversion is key to the dynamics and evolution of the boundary layer and a large gradient (e.g., temperature difference $\Delta \theta \sim 10 \mathrm{~K}$ in a thin layer $\sim 10 \mathrm{~m}$, which is comparable to the typical LES model vertical grid spacing $\Delta z$ ) challenges numerical approximations. Flow features become underresolved and turbulence model performance often degrades in this very stable regime.

However, the "inversion strength hypothesis" is inconsistent with results of "dry" (without water condensate) convective boundary layers (e.g., Schmidt and Schumann 1989; Sullivan et al. 1998; Sullivan and Patton 2011), which can also include sharp capping inversions. In particular, the dry convective LES runs of Sullivan and Patton (2011) with inversion $\Delta \theta \approx 6 \mathrm{~K}$ exhibit entrainment rate convergence for $\Delta z \leq 8 \mathrm{~m}$. The grid convergence criteria of Sullivan and Patton (2011) and Matheou and Chung (2014) imply grid convergence at about $\Delta z=10 \mathrm{~m}$. The underlying hypothesis of the present study is that merely the sharp capping inversion should not lead to poor model performance. Presently, the focus is on nocturnal nonprecipitating stratocumulus cloud decks, which is perhaps the simplest case with respect to physical processes including, in addition to stratified flow turbulent transport, vapor-liquid phase changes and longwave radiative transfer. Accordingly, the case of a nocturnal Sc corresponding to the first research flight (RF01) of the second Dynamics and Chemistry of Marine Stratocumulus (DYCOMS-II) field study (Stevens et al. 2003a) is simulated.

The present study aims to investigate three questions relating to the predictive skill of LES models in Sc simulations and the boundary layer physics:

- Why is grid convergence difficult to achieve?

- What parameters control the cloud cover and boundary layer turbulence in the LES?

- How does entrainment depend on numerical model and physical choices in the LES?

The questions are posed in the context of a specific LES model and turbulence closure that was shown to perform well in other boundary layer flows (Matheou and Chung 2014).

The methodology is centered around a parametric study that varies both numerical and physical properties of the system, primarily focusing on atmospheric physics perturbations rather than variations of parameterization parameters. This approach is a consequence of the working hypothesis that asserts that the modeling challenge is a consequence of the cloud physics. Accordingly, the present approach differs from the numerical experiments of Stevens et al. (2005), Pedersen et al. (2016), and Pressel et al. (2017) where an optimal model setup is sought by exploring different grid aspect ratios, numerical methods, and turbulence parameterizations. The present simulations are an extension of the simulations of Matheou and Chung (2014) where an identical numerical model setup is used to simulate a diverse set boundary layer types.

Moreover, the design of the present study offers insight into the Sc physics. For instance, it is often, confidently, stated that stratocumulus is driven by cloud-top radiative cooling (e.g., the first paragraph in Wood (2012)). But this simple (yet profound) statement raises several questions regarding the role of radiative forcing and the comparative roles of surface heat fluxes and wind shear in driving the mean and turbulent structure of Sc.

The quality of the LES results is evaluated with respect to 1) measurements from the observational campaign (Stevens et al. 2003a, 2005), 2) grid convergence of the flow statistics, and 3) the spectral energy distribution of velocity, temperature, and humidity. It is shown, for the first time, that a simulation that captures all three criteria, without employing any ad hoc model choices, is attainable.

The LES model and design of the parametric study are described in section 2. The results of the simulations are discussed in section 3. First, the results of the grid convergence study are presented for simulations with and without radiation. Model validation is also discussed in section 3a. Perturbation numerical experiments with respect to buoyancy and surface fluxes are presented in sections $3 \mathrm{~b}$ and $3 \mathrm{c}$. The effects of scalar advection discretization are discussed in section $3 \mathrm{~d}$. A detailed discussion of the entrainment rate is presented in section $3 \mathrm{e}$ and turbulence spectra are presented in section 3f. Finally, in the conclusion section, answers to the three main questions posed in the introduction section are considered.

\section{Methodology}

\section{a. Large-eddy simulation model}

The LES model formulation is typical for shallow convection studies. However, because model physics are altered in the parametric study, the governing equations 
are briefly documented. Additional details of the numerical approximation are described in appendix A. The LES model of Matheou and Chung (2014) is used. The Favre-filtered (density weighted) anelastic approximation of the Navier-Stokes equations (Ogura and Phillips 1962) is numerically integrated on an $f$ plane ( $\{$ zonal, meridional, vertical $\}=\left\{x_{1}, x_{2}, x_{3}\right\}=\{x, y, z\}$ ). The conservation equations for mass, momentum, liquid water potential temperature, and total water, neglecting resolved-scale viscous terms, are, respectively,

$$
\begin{gathered}
\frac{\partial \bar{\rho}_{0} \tilde{u}_{i}}{\partial x_{i}}= \\
\frac{\partial \bar{\rho}_{0} \tilde{u}_{i}}{\partial t}+\frac{\partial\left(\bar{\rho}_{0} \tilde{u}_{i} \tilde{u}_{j}\right)}{\partial x_{j}}= \\
-\theta_{0} \bar{\rho}_{0} \frac{\partial \bar{\pi}_{2}}{\partial x_{i}}+\delta_{i 3} b^{\prime} \\
-\varepsilon_{i j k} \bar{\rho}_{0} f_{j}\left(\tilde{u}_{k}-u_{g, k}\right)-\frac{\partial \tau_{i j}}{\partial x_{j}},
\end{gathered}
$$

$\frac{\partial \bar{\rho}_{0} \widetilde{\theta}_{l}}{\partial t}+\frac{\partial \bar{\rho}_{0} \widetilde{\theta}_{l} \tilde{u}_{j}}{\partial x_{j}}-D x_{3} \frac{\partial \bar{\rho}_{0} \widetilde{\theta}_{l}}{\partial x_{3}}=-\frac{1}{\pi c_{p}} \frac{\partial F_{\mathrm{rad}}}{\partial x_{3}}-\frac{\partial \sigma_{\theta, j}}{\partial x_{j}}$,

$\frac{\partial \bar{\rho}_{0} \widetilde{q}_{t}}{\partial t}+\frac{\partial \bar{\rho}_{0} \widetilde{q}_{t} \tilde{u}_{j}}{\partial x_{j}}-D x_{3} \frac{\partial \bar{\rho}_{0} \widetilde{q}_{t}}{\partial x_{3}}=-\frac{\partial \sigma_{q, j}}{\partial x_{j}}$.

The Favre-filtered variables are defined as $\tilde{\phi} \equiv \overline{\rho \phi} / \bar{\rho}$, where $\rho$ is the density and the overbar denotes a spatially filtered variable. The thermodynamic variables are decomposed into a constant potential temperature basic state, denoted by subscript 0 , and a dynamic component. Accordingly, $\theta_{0}$ is the constant basic-state potential temperature and $\rho_{0}(z)$ is the density. The Cartesian components of the velocity vector and geostrophic wind, are $u_{i}$ and $u_{g, i}$, respectively; $f=\left[0,0, f_{3}\right]$ is the Coriolis parameter; and $b^{\prime}$ the buoyancy perturbation with respect to the horizontal mean buoyancy. The subgridscale (SGS) terms $\tau_{i j}$ and $\sigma_{j}$ are estimated using the buoyancy adjusted stretched-vortex subgrid-scale model (Chung and Matheou 2014). The thermodynamic pressure, $p$, in each grid cell is computed from the sum of the basic state Exner function, $\pi_{0}(z)$, plus a contribution due to the deviation of the horizontal mean from the basic state, $\pi_{1}(t, z)$, and the dynamic part $\pi_{2}(t, x, y, z)$, which enforces the anelastic constraint Eq. (1),

$$
\pi=\frac{\pi_{0}+\pi_{1}+\pi_{2}}{c_{p}}=\frac{T}{\theta}=\left(\frac{p}{p_{\text {ref }}}\right)^{R / c_{p}},
$$

where $R=287.04 \mathrm{~J} \mathrm{~kg}^{-1} \mathrm{~K}^{-1}$ is the gas constant of dry air, and $c_{p}=1005 \mathrm{~J} \mathrm{~kg}^{-1} \mathrm{~K}^{-1}$ the specific heat capacity.
The effect of the large-scale environment is included in the equations for $\theta_{l}$ and $q_{t}$ through the subsidence terms and the geostrophic wind. The uniform largescale horizontal divergence is denoted by $D$. The liquid water potential temperature equation includes radiative heating and cooling through the net radiative flux $F_{\text {rad }}$ divergence.

The subgrid condensation scheme is "all or nothing" (i.e., no partially saturated air in each grid cell is assumed). The thermodynamic state at the grid cell center is used to classify each grid cell as saturated or not and determine the corresponding thermodynamic coefficients for all variables, including those residing at the cell's vertices. All water condensate is suspended, thus no drizzle/precipitation is allowed.

\section{b. Parametric study}

\section{1) BASE MODEL SETUP AND PERTURBATION NUMERICAL EXPERIMENTS}

The simulations are based on the LES setup of the nocturnal Sc observed during the first research flight (RF01) of the second Dynamics and Chemistry of Marine Stratocumulus (DYCOMS-II) campaign (Stevens et al. 2003a) and the base setup follows Stevens et al. (2005). Even though the base setup is likely the most often simulated Sc case and it is comparatively simple (does not include precipitation, diurnal cycle, cloud-aerosol interactions), the case is a challenging simulation. The low liquid water path (LWP) and the (purposely chosen) structure of the boundary layer corresponding to coupled layer that is close to becoming decoupled requires skillful models.

All simulations have a doubly periodic computational domain in the horizontal directions and identical initial $q_{t}$ and $\theta$ profiles corresponding to a mixed layer of $z_{i}=840-\mathrm{m}$ depth. The computational domain size is $5.12^{2} \times 1.5 \mathrm{~km}$ for all runs. A Rayleigh damping layer is applied above $1.2 \mathrm{~km}$, to limit gravity wave reflection. Following Stevens et al. (2005), the simulations are run for $4 \mathrm{~h}$ after initialization, except the highestresolution cases that were only run up to $t=2 \mathrm{~h}$ to conserve computing time. As shown in appendix B, the results at $t=2 \mathrm{~h}$ are representative of the boundary layer structure.

In the base case the large-scale horizontal divergence is $D=3.75 \times 10^{-6} \mathrm{~s}^{-1}$. Radiation parameterization and the buoyancy definition are discussed in the following two subsections.

Seven parameters are varied in the LES: the grid spacing, geostrophic wind, divergence, radiation parameterization, buoyancy formulation, surface fluxes, and the scalar advection numerical method. The parameter 
TABLE 1. Links between the study's questions and the model parameters. Table entries correspond to the simulation cases.

\begin{tabular}{lcccc}
\hline & \multicolumn{3}{c}{ Parameter } \\
\cline { 2 - 5 } \multicolumn{1}{c}{ Objective } & $\Delta x$ & $F_{\text {rad }}$ & Buoyancy & Surface flux \\
\hline Grid convergence & $\mathrm{A}$ & $\mathrm{B}$ & $\mathrm{C}$ & Numerics \\
Entrainment & $\mathrm{A}$ & $\mathrm{B}$ & $\mathrm{C}$ & $\mathrm{D}, \mathrm{E}, \mathrm{F}, \mathrm{G}$ \\
Cloud amount and turbulence & $\mathrm{A}$ & $\mathrm{B}$ & $\mathrm{C}$ & $\mathrm{H}$ \\
\hline
\end{tabular}

variations are grouped in Table 1 into three broad objectives labeled: "grid convergence," "entrainment," and "cloud amount and turbulence." The objectives correspond to the main questions posed in the introduction. A total of 16 LES runs were carried out and are summarized in Table 2 and Fig. 1. Each run is labeled as $X n$, where $X$ is a letter corresponding to the perturbation experiment (see Fig. 1) and $n$ a number that denotes the grid resolution.

\section{2) GRID RESOLUTION}

Five grid resolutions are used $\Delta x=1.25,2.5,5,10$, and $20 \mathrm{~m}$. All grids are uniform and isotropic (i.e., $\Delta x=\Delta y=$ $\Delta z$ ). Runs with $\Delta x=1.25 \mathrm{~m}$ are labeled $X 1, \Delta x=2.5 \mathrm{~m}$ labeled $X 2$, etc. The highest-resolution simulations have 20 billion grid cells, thus are computationally challenging. Series A runs (Table 2) follow the configuration of Stevens et al. (2005) and explore the effects of grid resolution.

\section{3) RADiATIVE FLUX}

The net longwave radiative flux is parameterized as (Stevens et al. 2005)

$$
\begin{aligned}
F_{\mathrm{rad}}(t, x, y, z)= & F_{0} e^{-Q(z, \infty)}+F_{1} e^{-Q(0, z)} \\
& +\rho\left(z_{i}\right) c_{p} D \alpha_{z}\left[\left(z-z_{i}\right)^{4 / 3} / 4+z_{i}\left(z-z_{i}\right)^{1 / 3}\right],
\end{aligned}
$$

where $z_{i}(t, x, y)$ is the column-wise inversion height,

$$
Q\left(z_{1}, z_{2}\right)=\kappa \int_{z_{1}}^{z_{2}} \rho r_{l}(t, x, y, z) d z
$$

and $r_{l}$ is the liquid water mixing ratio. The values of the constants are $F_{0}=70 \mathrm{~W} \mathrm{~m}^{-2}, F_{1}=22 \mathrm{~W} \mathrm{~m}^{-2}, \kappa=$ $85 \mathrm{~m}^{2} \mathrm{~kg}^{-1}$, and $\alpha_{z}=1 \mathrm{~m}^{-4 / 3}$. The radiation parameterization causes strong cooling in a thin layer $(\sim 10 \mathrm{~m})$ at the cloud top and small heating near the cloud base. The radiation flux is calculated at each model time step column-wise.

A set of runs, series B, was carried out without radiation by setting $F_{\text {rad }}=0$ in the model to explore the impact of cloud-top radiative cooling. Radiation is expected to have a strong impact on the turbulent flow structure because cloud-top cooling is a significant source of buoyancy. Moreover, because of the exponential

TABLE 2. Summary of the cases simulated. The grid spacing is denoted by $\Delta x, N_{x}=N_{y}$ and $N_{z}$ are the number of horizontal and vertical grid points, respectively, "Wind" corresponds to forcing with the geostrophic wind $\mathbf{u}_{g}$ or no wind (i.e., no mean surface shear). Simulations without radiation have null radiative flux at all model levels, denoted by $F_{\text {rad }}=0$. A modified buoyancy variable [Eq. (10)] is used in case

\begin{tabular}{|c|c|c|c|c|c|c|c|c|c|}
\hline Run & $\Delta x$ & $N_{x}$ & $N_{z}$ & Wind & $D \times 10^{-6}\left(\mathrm{~s}^{-1}\right)$ & Radiation & Buoyancy & Surface fluxes & Scalar advection \\
\hline A1 & 1.25 & 4096 & 1200 & $\mathbf{u}_{g}$ & 3.75 & Eq. (6) & Multiphase & Prescribed & QUICK \\
\hline $\mathrm{A} 2$ & 2.5 & 2048 & 600 & $\mathbf{u}_{g}$ & 3.75 & Eq. (6) & Multiphase & Prescribed & QUICK \\
\hline A3 & 5 & 1024 & 300 & $\mathbf{u}_{g}$ & 3.75 & Eq. (6) & Multiphase & Prescribed & QUICK \\
\hline A4 & 10 & 512 & 150 & $\mathbf{u}_{g}$ & 3.75 & Eq. (6) & Multiphase & Prescribed & QUICK \\
\hline A5 & 20 & 256 & 75 & $\mathbf{u}_{g}$ & 3.75 & Eq. (6) & Multiphase & Prescribed & QUICK \\
\hline B1 & 1.25 & 4096 & 1200 & $\mathbf{u}_{g}$ & 3.75 & $F_{\text {rad }}=0$ & Multiphase & Prescribed & QUICK \\
\hline B2 & 2.5 & 2048 & 600 & $\mathbf{u}_{g}$ & 3.75 & $F_{\mathrm{rad}}=0$ & Multiphase & Prescribed & QUICK \\
\hline B3 & 5 & 1024 & 300 & $\mathbf{u}_{g}^{\delta}$ & 3.75 & $F_{\mathrm{rad}}=0$ & Multiphase & Prescribed & QUICK \\
\hline B4 & 10 & 512 & 150 & $\mathbf{u}_{g}^{\delta}$ & 3.75 & $F_{\text {rad }}=0$ & Multiphase & Prescribed & QUICK \\
\hline B5 & 20 & 256 & 75 & $\stackrel{0}{\mathbf{u}_{g}}$ & 3.75 & $F_{\text {rad }}=0$ & Multiphase & Prescribed & QUICK \\
\hline C3 & 5 & 1024 & 300 & $\mathbf{u}_{g}$ & 3.75 & Eq. (6) & Modified & Prescribed & QUICK \\
\hline D3 & 5 & 1024 & 300 & $\mathbf{u}_{g}$ & 0 & Eq. (6) & Multiphase & $\overline{w \theta}_{v}=0$ & QUICK \\
\hline E3 & 5 & 1024 & 300 & $0^{8}$ & 0 & Eq. (6) & Multiphase & $\overline{w \theta}_{v}=0$ & QUICK \\
\hline F3 & 5 & 1024 & 300 & $\mathbf{u}_{g}$ & 0 & $F_{\mathrm{rad}}=0$ & Multiphase & $\overline{w \theta}_{v}=0$ & QUICK \\
\hline G1 & 1.25 & 4096 & 1200 & $\mathbf{u}_{g}$ & 3.75 & Eq. (6) & Multiphase & MOST & QUICK \\
\hline $\mathrm{H} 3$ & 5 & 1024 & 300 & $\mathbf{u}_{g}$ & 3.75 & Eq. (6) & Multiphase & Prescribed & Centered \\
\hline
\end{tabular}
C3. Surface sensible and latent heat fluxes are either spatially uniform, denoted by "prescribed," dynamically estimated at each grid cell using MOST, or zero $\overline{w \theta}_{v}=0$, For all runs $\Delta x=\Delta y=\Delta z$. 
A

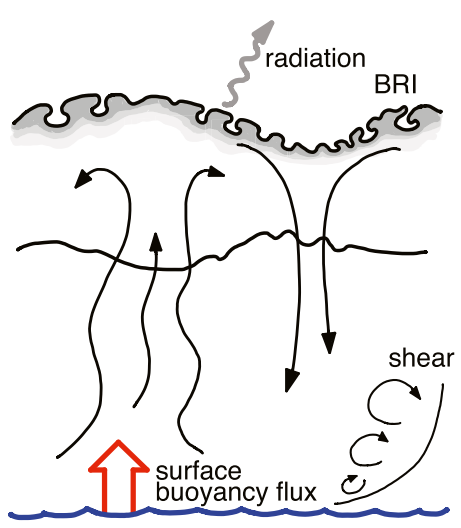

D

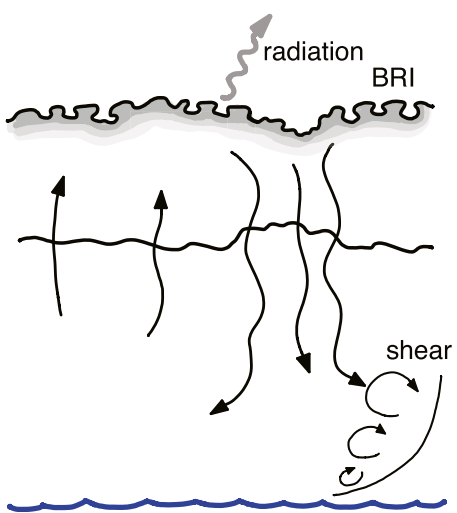

B

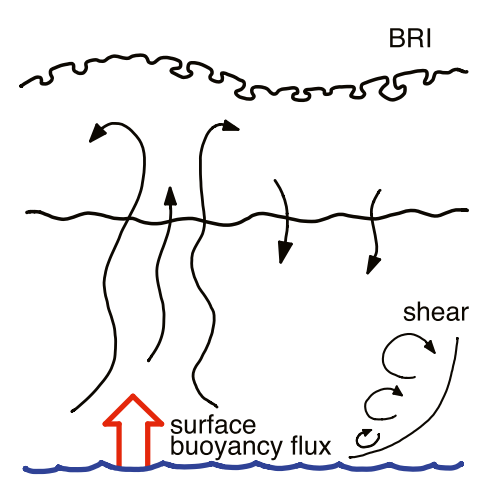

E

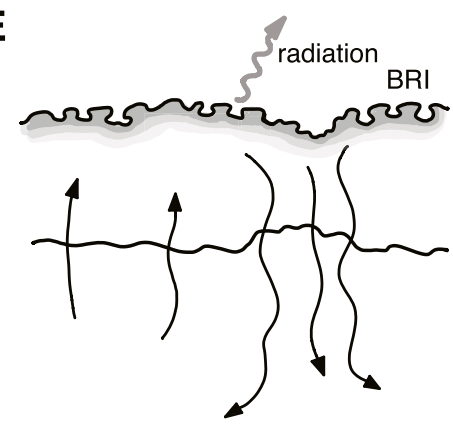

C

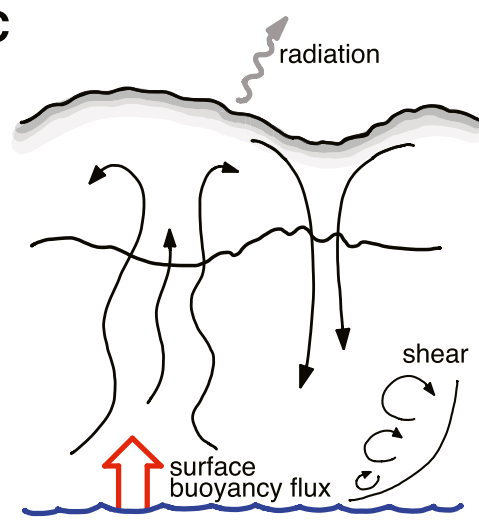

$\mathbf{F}$

BRI
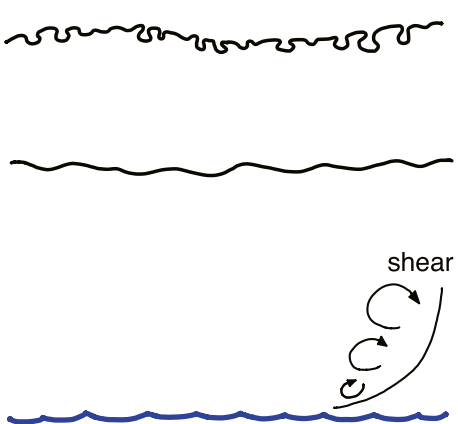

FIG. 1. Physical processes in the simulation cases. The top left label corresponds to the case (see Table 2). The shading of the cloud top denotes runs with radiation. A corrugated cloud top represents the buoyancy reversal instability (BRI). The cloud-top height variations are exaggerated to show the upward deformation of the inversion surface when updrafts impinge on the free troposphere and the downward deformation in the region of entrainment events.

dependence of $F_{\text {rad }}$ on LWP, radiation strongly links the thermodynamic structure with momentum leading to feedbacks between cloud amount and turbulence generation.

\section{4) BUOYANCY}

One of the main difficulties in LES of the DYCOMSII RF01 case is the dissipation of cloud and associated reduction of LWP. The cloud dissipation can be a model artifact caused, for instance, by excessive cloud-top mixing with the drier free troposphere, but also because of cloud physics, such as the cloud-top entrainment instability (CTEI) (Deardorff 1980; Randall 1980). To examine the effects of the two-phase flow in the simulations a modified definition of buoyancy is used.

In the standard LES model, buoyancy is defined proportional to deviations of virtual potential temperature $\theta_{v}$ from its instantaneous horizontal average $\left\langle\tilde{\theta}_{v}\right\rangle$,

$$
b^{\prime}=g \bar{\rho}_{0} \frac{\tilde{\theta}_{v}-\left\langle\tilde{\theta}_{v}\right\rangle}{\theta_{0}} .
$$

The virtual potential temperature is

$$
\theta_{v}=\theta\left[1+\left(R_{m} / R-1\right) r-r_{l}\right],
$$

where $r$ is the water vapor mixing ratio and $R_{m}=$ $461.5 \mathrm{~J} \mathrm{~kg}^{-1} \mathrm{~K}^{-1}$ the gas constant of water vapor.

In run $\mathrm{C} 3, \theta_{v}$ is modified

$$
\theta_{v, \text { mod }}=\theta_{l}\left[1+\left(R_{m} / R-1\right) q_{t}\right]
$$

The modified $\theta_{v}$ is similar to its definition for air without condensate with $\theta_{l}$ and $q_{t}$ in the place of $\theta$ and $r$. Effectively, $\theta_{v \text {,mod }}$ makes the buoyancy term behave as if the boundary layer is a dry convective one by excluding latent heat exchange. Accordingly, in run $\mathrm{C} 3$ the turbulent virtual potential temperature flux $\overline{w \theta_{v}}$ is calculated from the $\overline{w \theta_{l}}$ and $\overline{w q_{t}}$ fluxes by assuming unsaturated air (Cuijpers and Duynkerke 1993).

\section{5) SURFACE FLUXES AND SHEAR}

In the base case, that is, the model setup of Stevens et al. (2005), the surface sensible and latent heat fluxes 
are spatially uniform and constant in time and their values are 15 and $115 \mathrm{~W} \mathrm{~m}^{-2}$, respectively. The momentum fluxes are dynamically computed in each grid cell using Monin-Obukhov similarity theory (MOST). Cases $\mathrm{A}, \mathrm{B}$, and $\mathrm{H}$ use the surface flux parameterization of Stevens et al. (2005).

A key feature of stratocumulus is the generation of convective motions by cloud-top radiative cooling (Stevens 2005; Wood 2012). Two main sources of buoyancy are present in the base case: surface heat fluxes and cloud-top cooling. In Cases D, E, and F the surface buoyancy flux is set to zero (by imposing zero sensible and latent heat fluxes). Thus, only cloud-top generated convective motions are present in Cases $\mathrm{D}$, $\mathrm{E}$, and $\mathrm{F}$, whereas Cases $\mathrm{A}, \mathrm{B}$, and $\mathrm{C}$ include surface buoyancy flux. Flow visualizations of base case show strong surface-generated convection (Matheou et al. 2017). Thus, a null surface buoyancy flux is expected to have a large impact.

Because the growth rate of the boundary layer is significantly reduced when surface heat fluxes are zero, the large-scale divergence is set to zero in cases $\mathrm{D}, \mathrm{E}$, and F to avoid a decreasing boundary layer depth with time. In the base case, subsidence approximately balances the entrainment rate and the boundary layer depth slightly increases during the simulation.

The cases with variations of the surface flux $(\mathrm{D}, \mathrm{E}$, and F) examine the impact of different turbulence generation mechanisms.

Case D is identical to the base case but with sensible and latent heat fluxes set to zero. Surface momentum fluxes are computed using MOST. Shear near the surface and buoyancy from cloud-top radiative cooling are the main turbulence sources.

Case $\mathrm{E}$ is similar to case $\mathrm{D}$ but without the mean shear turbulence production. This is accomplished by setting the geostrophic wind and initial wind profile to zero.

Case $\mathrm{F}$ is similar to case $\mathrm{D}$ but without radiation. Thus, in case $\mathrm{F}$ there is no buoyancy-driven turbulence and surface shear primarily drives turbulence.

The present approach differs from the usual study of the relative contribution of terms of the TKE or the turbulent fluxes equations (e.g., Heinze et al. 2015) because a physical process decomposition is currently employed. For instance, the examination of the secondmoment budgets can quantify the contribution of the local shear production, but from a single run it is difficult to infer the contributions of surface shear and shear generated within the updrafts and downdrafts.

Case $\mathrm{G}$ differs from the base case by using MOST to estimate both sensible and latent surface heat fluxes, in addition to momentum flux, using $292.5 \mathrm{~K}$ for the sea surface temperature (Stevens et al. 2005) and the
Charnock (1955) parameterization for the roughness length. As shown in Matheou and Chung (2014), using MOST to estimate the surface fluxes results in a similar flow as the base case with uniform heat fluxes. Case $G$ is used to investigate spectra and using a dynamic surface flux that adjusts to the local near-surface flow is expected to yield more representative spectra.

The grid resolution for cases $\mathrm{C}, \mathrm{D}, \mathrm{E}$, and F is $\Delta x=5 \mathrm{~m}$. The flow statistics are likely not fully converged at $\Delta x=5 \mathrm{~m}$ based on the results of the grid resolution study (see section 3a). However, the model results are representative of the overall flow structure and conclusions based on relative differences are expected to be reliable.

\section{6) SCALAR ADVECTION DISCRETIZATION}

Many LES models use positive definite scalar advection schemes (e.g., Smolarkiewicz and Margolin 1998; Stevens et al. 2005; Sullivan and Patton 2011), which preserve the local bounds of scalar quantities. Such methods introduce numerical dissipation, which can overpower and indiscriminately suppress the SGS model physics. Conversely, nondissipative schemes can produce numerical artifacts in the form of spurious $\theta_{l}$ and $q_{t}$ under and overshoots above the Sc inversion due to dispersive errors (Matheou and Dimotakis 2016). This type of numerical error affects the mean profiles (Matheou and Chung 2014, their Fig. 16) and can potentially impact the global boundary layer dynamics because of the contribution of temperature and humidity to buoyancy.

Case $\mathrm{H} 3$ assesses the impact of numerical errors from nondissipative scalar advection schemes and uses the fourth-order centered scheme of Morinishi et al. (1998) for $\theta_{l}$ and $q_{t}$. All other cases use Quadratic Upstream Interpolation for Convective Kinematics (QUICK; Leonard 1979), a linear upwinding scheme. QUICK does not enforce monotonicity (positive definiteness) of the advected scalar fields and it is less dissipative than monotone schemes (e.g., Matheou and Dimotakis 2016). Further details of the scalar advection discretization are discussed in appendix A.

\section{Simulations}

\section{a. Grid convergence and cloud-top radiative cooling feedback}

The time traces (Fig. 2) and profiles (Fig. 3) for the base case show that grid convergence of high-order statistics is challenging and that not all boundary layer statistics exhibit the same convergence properties. In all cases, profiles are instantaneous horizontal averages 

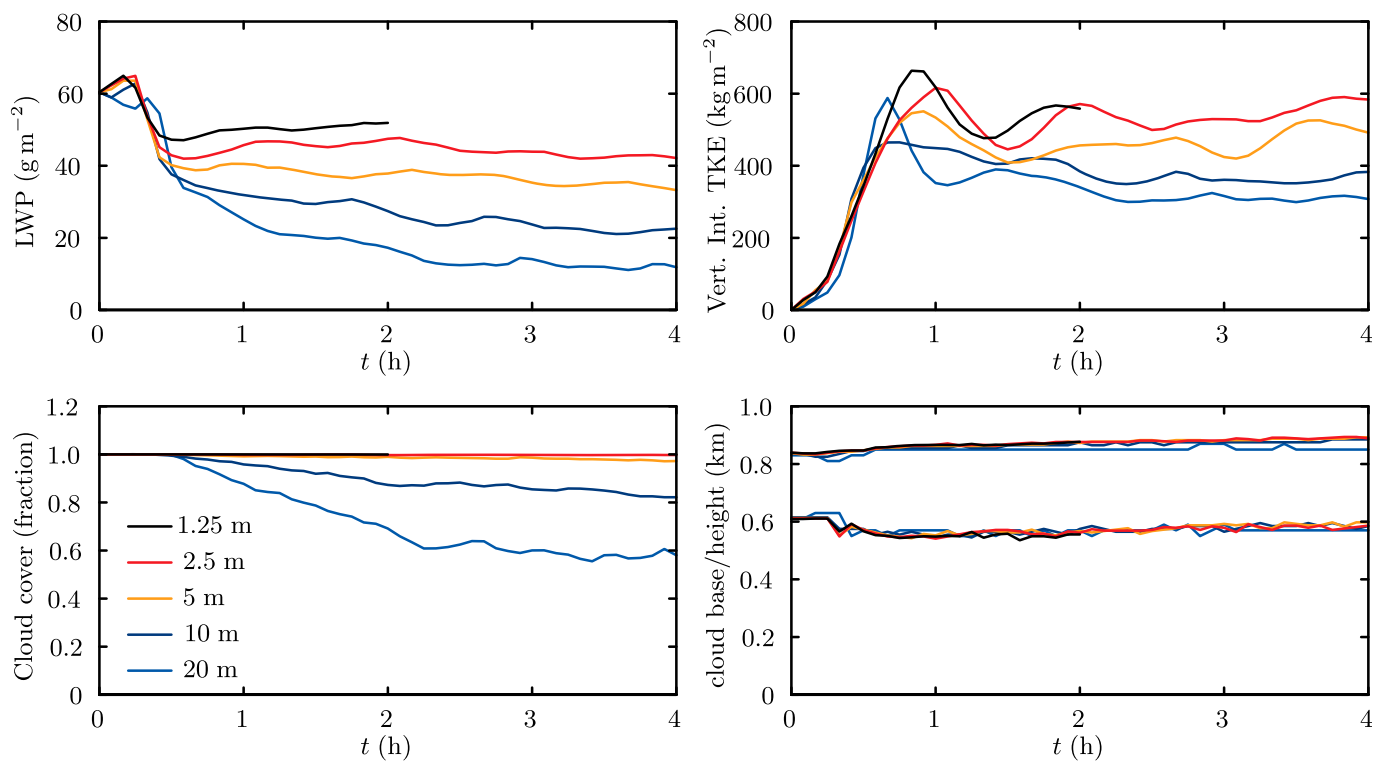

FIG. 2. Time traces of liquid water path, vertically integrated turbulent kinetic energy, cloud cover, and cloud-top and cloud-base height for series A runs. All runs have identical model configuration but grid resolution varies as shown in the legend.

(no time averaging). Even though resolution is varied by a factor of 16 , with a corresponding computational cost factor of $16^{4}$, the mean profiles of the conserved variables, Eqs. (2)-(4), do not significantly depend on $\Delta x$. Moreover, with the exception of the coarsest grid
$\Delta x=20 \mathrm{~m}$, cloud-base and cloud-top height, and entrainment rate are resolution independent (the details of the entrainment rate grid convergence are further discussed in section 3e). The subgrid fractions of the turbulent fluxes are discussed in appendix C. All series
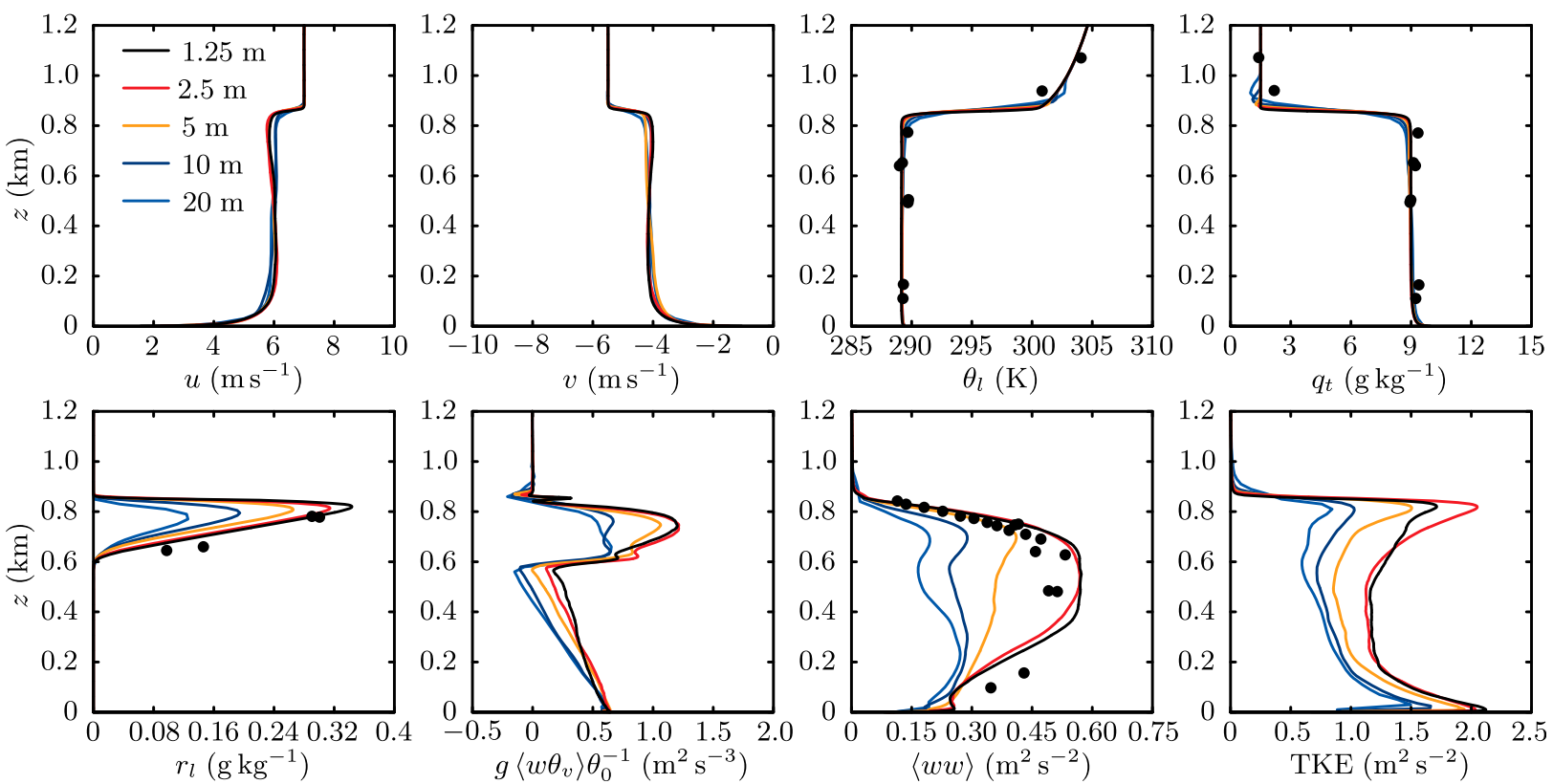

FIG. 3. Profiles of zonal wind $u$ and meridional wind $v$, liquid water potential temperature $\theta_{l}$, total water mixing ratio $q_{t}$, liquid water mixing ratio $r_{l}$, buoyancy flux $g\left\langle w \theta_{v}\right\rangle \theta_{0}^{-1}$, vertical velocity variance $\langle w w\rangle$, and turbulent kinetic energy (TKE) at $t=2 \mathrm{~h}$ (no time averaging) for series A runs. Turbulence statistics include the subgrid-scale contribution. All runs have identical model configuration but grid resolution varies as shown in the legend. Circles denote observations from Stevens et al. (2005). 

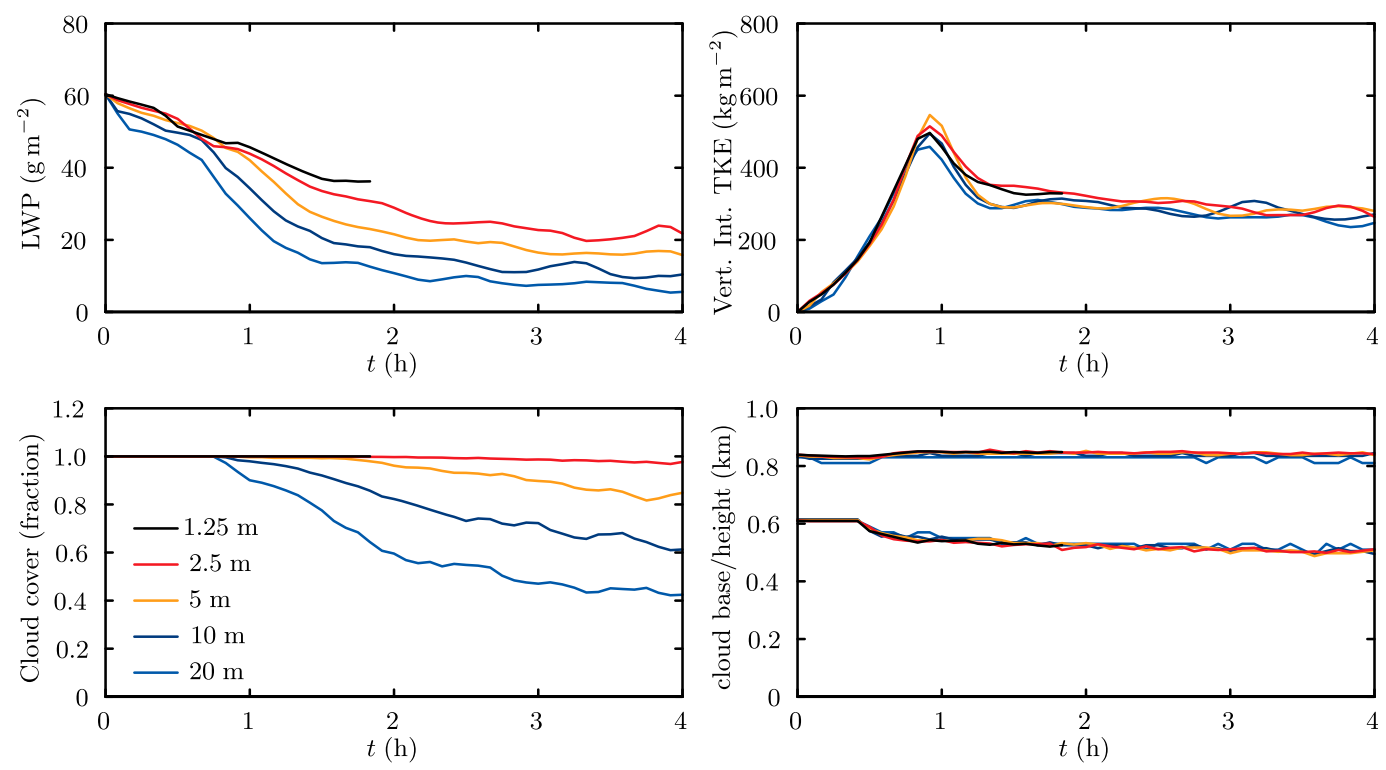

FIG. 4. Time traces of liquid water path, vertically integrated turbulent kinetic energy, cloud cover, and cloud-top and cloud-base height for series B runs. All runs have identical model configuration but grid resolution varies as shown in the legend. Series B configuration is similar to series A shown in Fig. 2 but does not include radiation.

A simulations show a well-mixed layer for $u, v, \theta_{l}$, and $q_{t}$ capped by an inversion.

All simulations have an initial period of adjustment or "spin up" in the first hour of the run during which three-dimensional turbulence develops. The established convection causes horizontal variability within the cloud with updrafts saturating at different heights causing a lowering of the cloud-base height and a LWP decrease with respect to the initial condition (Matheou 2018).

The amount of cloud liquid and turbulent fluxes show significant variations with respect to $\Delta x$. As grid resolution becomes coarser, $r_{l}$ and the magnitude of $\langle w w\rangle$, and turbulent kinetic energy decrease. Moreover, the buoyancy flux profiles show the turbulence structure of convection tending toward a decoupled boundary layer when $\Delta x$ increases with the buoyancy flux attaining negative values near cloud base.

The statistics for case A shown in Figs. 2 and 3, except $r_{l}$ and LWP, are essentially identical at the two highest resolutions, $\Delta x=2.5$ and $1.25 \mathrm{~m}$, and show good agreement with observations, especially the $\langle w w\rangle$ profiles. The $\langle w w\rangle$ profiles are somewhat larger than the observations, but this is likely because the comparison is at $t=2 \mathrm{~h}$ rather than $t=4 \mathrm{~h}$ as in Stevens et al. (2005). As shown in appendix $\mathrm{B},\langle w w\rangle$ decreases with time. Moreover, some of the differences between the LES and observations can be because of the approximations of the physical parameterizations, such as idealized radiation and no cloud droplet sedimentation. Both $r_{l}(z)$ and LWP appear to be converging with respect to $\Delta x$ with the two highest resolutions having $8 \%$ difference in LWP. The two coarsest grids $\Delta x \geq 10 \mathrm{~m}$ have small LWP and $\$ 100 \%$ cloud cover, where cloud cover is defined as the fraction of model columns with $r_{l}(x, y, z)>10^{-5} \mathrm{~kg} \mathrm{~kg}^{-1}$ at any level.

Overall, the grid convergence study of the base case (series A runs) suggests robust model performance with respect to the mean profiles and entrainment. The convergence rate of cloud liquid and the turbulent fluxes is slower requiring finer grid resolutions.

The time traces (Fig. 4) and profiles (Fig. 5) of series B runs, which do not include radiation, show grid independent turbulent fluxes and TKE. However, the cloud liquid shows similar variation with $\Delta x$ as the series A runs. The large variations of $r_{l}$, and consequently LWP, are because $r_{l}$ is diagnosed from $q_{t}$ in the model, thus about $0.5 \%$ error in $q_{t}$ corresponds to a $20 \%$ error in $r_{l}$, assuming negligible errors in temperature. In the runs without radiation (series B), the variation of $r_{l}$ with $\Delta x$ has small effect on the boundary layer turbulence and appears to mostly affect $\langle w w\rangle$ in the cloud layer, likely because of latent heat release of condensing rising thermals and buoyancy reversal when cloudy-clear air mixes below the inversion.

In the base case, the radiation flux depends exponentially on $r_{l}$ [Eq. (6)], thus any $r_{l}$ errors are amplified creating a strong feedback between $r_{l}$ and turbulence generation in the boundary layer. Comparison of the TKE traces and profiles, and turbulent fluxes between series $\mathrm{A}$ and $\mathrm{B}$ suggests the contribution of radiative cooling. A comparison of Figs. 3 and 5 shows that 

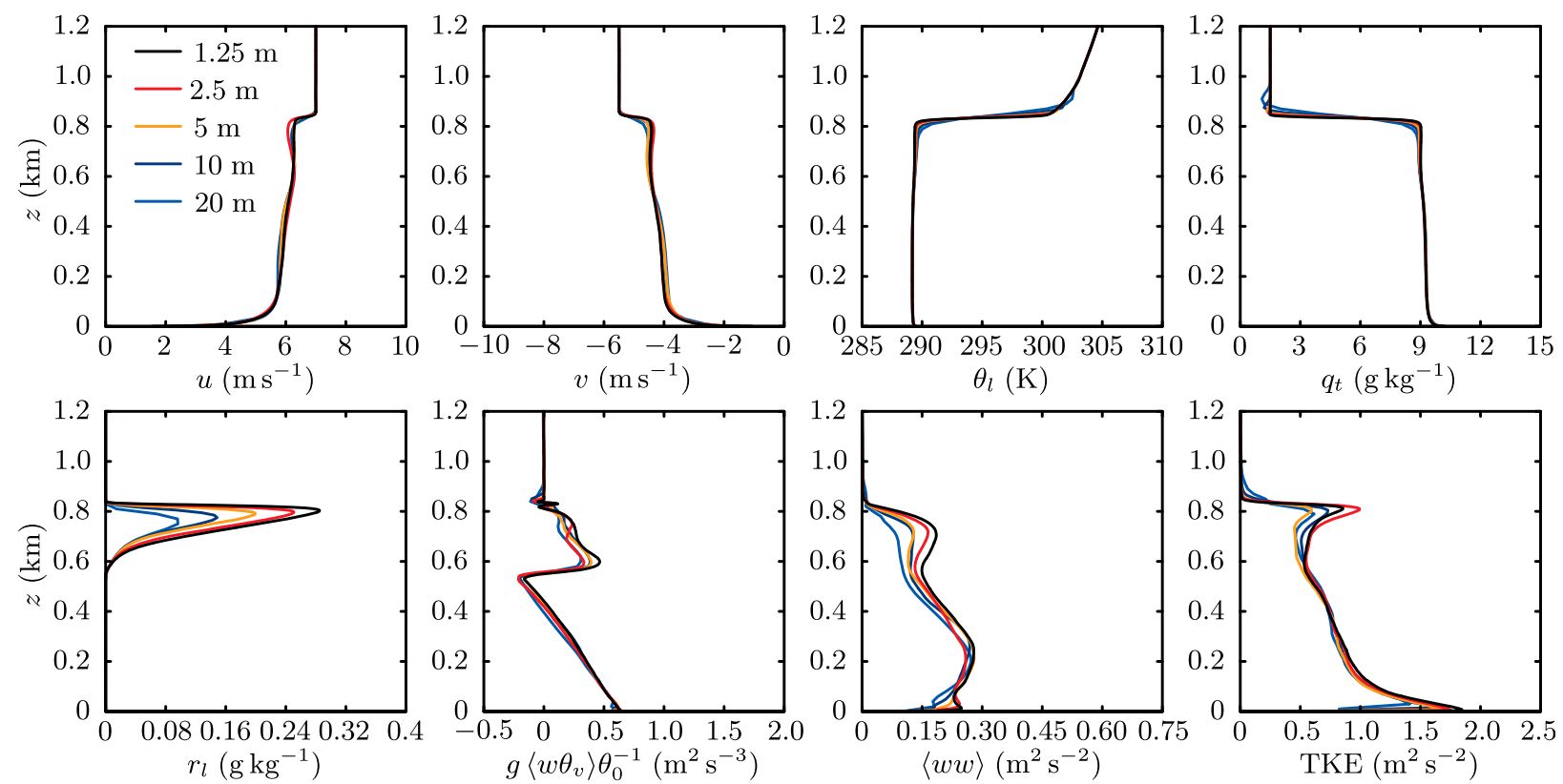

FIG. 5. Profiles of zonal wind $u$ and meridional wind $v$, liquid water potential temperature $\theta_{l}$, total water mixing ratio $q_{t}$, liquid water mixing ratio $r_{l}$, buoyancy flux $g\left\langle w \theta_{v}\right\rangle \theta_{0}^{-1}$, vertical velocity variance $\langle w w\rangle$, and turbulent kinetic energy (TKE) at $t=2 \mathrm{~h}$ (no time averaging) or series B runs. Turbulence statistics include the subgrid-scale contribution. All runs have identical model configuration but grid resolution varies as shown in the legend.

radiative cooling contributes about half the TKE in the upper part $(z>600 \mathrm{~m})$ of the boundary layer and about $2 / 3$ of the vertical velocity variance. Essentially, the turbulent flux profiles of Fig. 3 span the range between no radiation and a converged LES with radiation.

\section{b. Buoyancy}

Case C3 aims to (artificially) decouple buoyancy from latent heat exchange effects. Case C3 behaves similar to a dry convective boundary layer with the addition of the cloud-top buoyancy source because of radiative cooling. Thus, it is a "double" convective boundary layer driven by buoyancy both from the bottom and top.

The comparison of the time traces (Fig. 6) and profiles (Fig. 7) between cases $\mathrm{C} 3$ and $\mathrm{A} 3$ shows large differences, comparable to those between cases A and B. As shown in the cloud-top trace, case $\mathrm{C} 3$ entrains less than A3, resulting in a cooler and moister boundary with an increasing LWP and decreasing cloud-base height. Consequently, the cloud-top radiative cooling and the resulting buoyancy flux increase leading to a more energetic boundary layer (i.e., larger TKE and $\langle w w\rangle$ ).

Interestingly, the more vigorous turbulence of case C3 does not result in a higher entrainment rate compared to case A3. Since case C3 does not include the dynamics of buoyancy reversal and CTEI, the entrainment reduction suggests that these mechanisms contribute significantly to the entrainment rate.

\section{c. Surface fluxes}

Figures 8 and 9 compare time traces and profiles at $t=$ $4 \mathrm{~h}$ between the three cases with no sensible and latent heat surface fluxes (D3, E3, and F3).

For the short duration of these runs up to $t=4 \mathrm{~h}$, the two cases with a convective component (i.e., cloud-top radiative cooling, D3 and E3), appear to maintain the cloud cover and LWP, whereas the cloud is dissipating for case F3, which is only driven by surface shear. Since there is no moisture source in cases D-F the cloud will eventually dissipate because of entrainment of warmer and dryer air into the boundary layer.

The TKE traces in Fig. 8 and profiles in Fig. 9 suggest that, for the present case, surface-driven convection, radiative-driven convection, and surface shear contribute about equally to the entire TKE. This argument is qualitative, since the system is nonlinear.

The main contribution to $\langle w w\rangle$ near the surface is shear and cloud-top radiative cooling in the upper half of the boundary layer. Even though surface-emanating convection is not present in cases D3 and E3, $\langle w w\rangle$ is comparable to the base case, likely because negatively buoyant plumes emanating from the top of the boundary layer extend through the entire depth of the layer to form convective circulations.

The buoyancy flux profiles (Fig. 9) are similar in the cloud layer for cases A3, D3, and F3, implying that, 

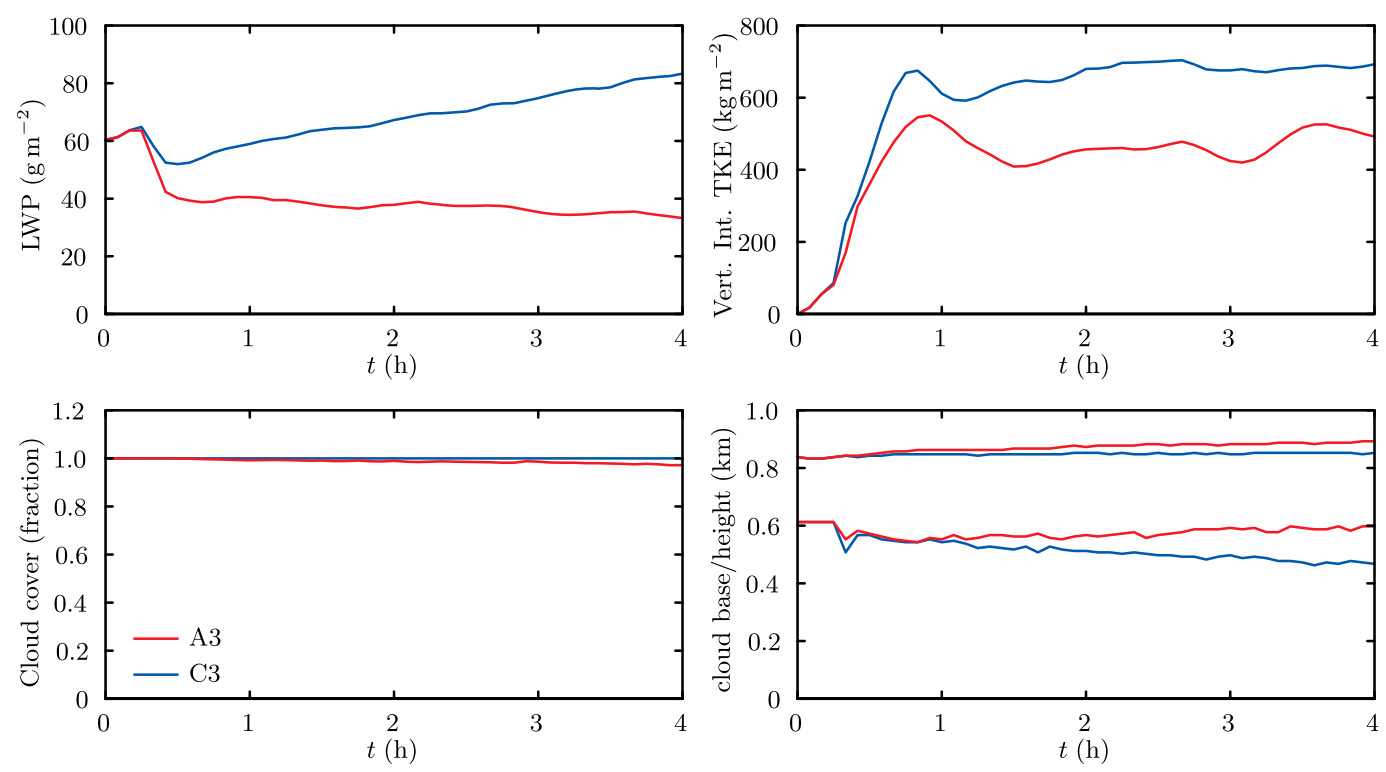

FIG. 6. Time traces of liquid water path, vertically integrated turbulent kinetic energy, cloud cover, and cloud-top and cloud-base height for cases A3 and C3. In case C3 buoyancy is modified and does not include the effects of two-phase flow.

perhaps as expected, this part of the $\left\langle w \theta_{v}\right\rangle$ profile depends only on radiative cooling.

\section{d. Scalar advection discretization}

In most previous simulations with the present LES model (e.g., Matheou and Chung 2014; Matheou 2016) the scalar advection scheme is nondissipative, which ensures that all dissipation is physical since it is provided by the SGS model. The nondissipative scheme generally performs well but in some cases numerical artifacts are present, typically near strong inversions $(\Delta \theta \sim 10 \mathrm{~K})$. The combination of two factors 1$)$ high $\theta_{l}$ and $q_{t}$ gradients and 2) the strong stability lead to large dispersive errors, thus $\theta_{l}$ and $q_{t}$ can attain values
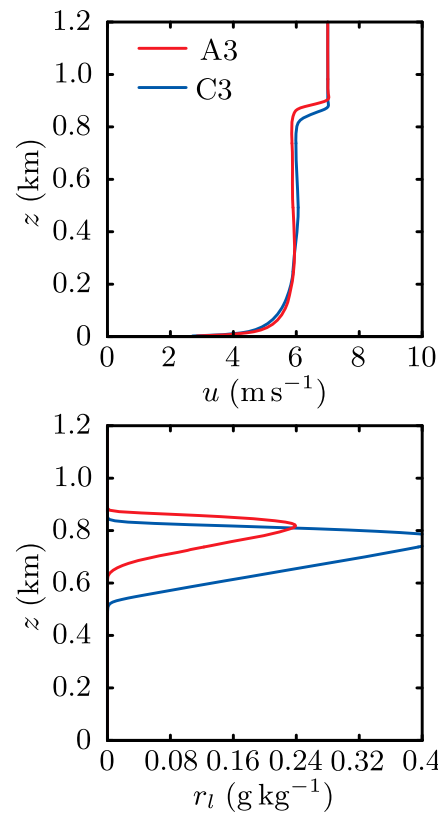
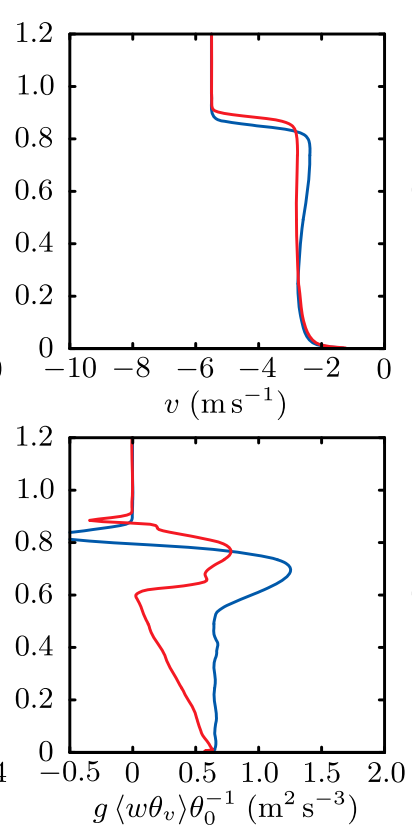
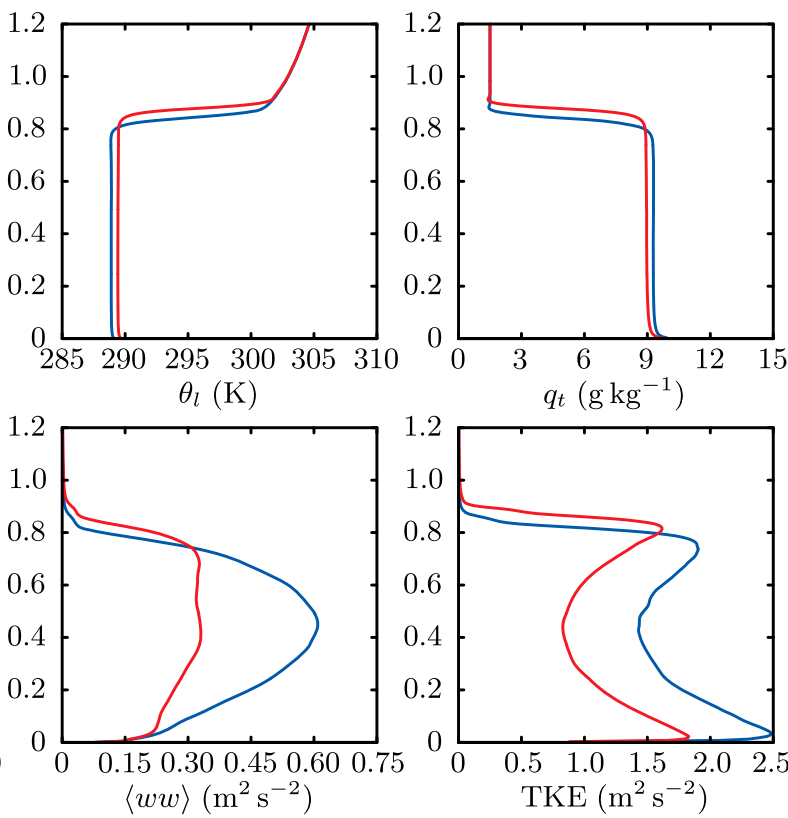

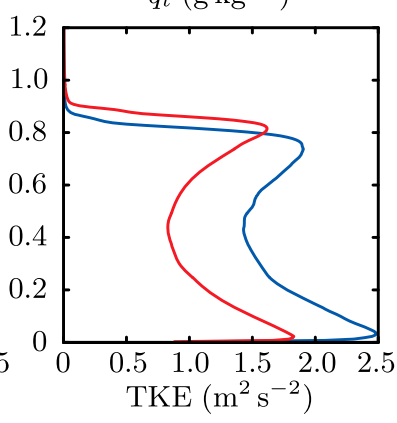

FIG. 7. Profiles of zonal wind $u$ and meridional wind $v$, liquid water potential temperature $\theta_{l}$, total water mixing ratio $q_{t}$, liquid water mixing ratio $r_{l}$, buoyancy flux $g\left\langle w \theta_{v}\right\rangle \theta_{0}^{-1}$, vertical velocity variance $\langle w w\rangle$, and turbulent kinetic energy (TKE) at $t=4 \mathrm{~h}$ (no time averaging) for cases A3 and C3. Turbulence statistics include the subgrid-scale contribution. 

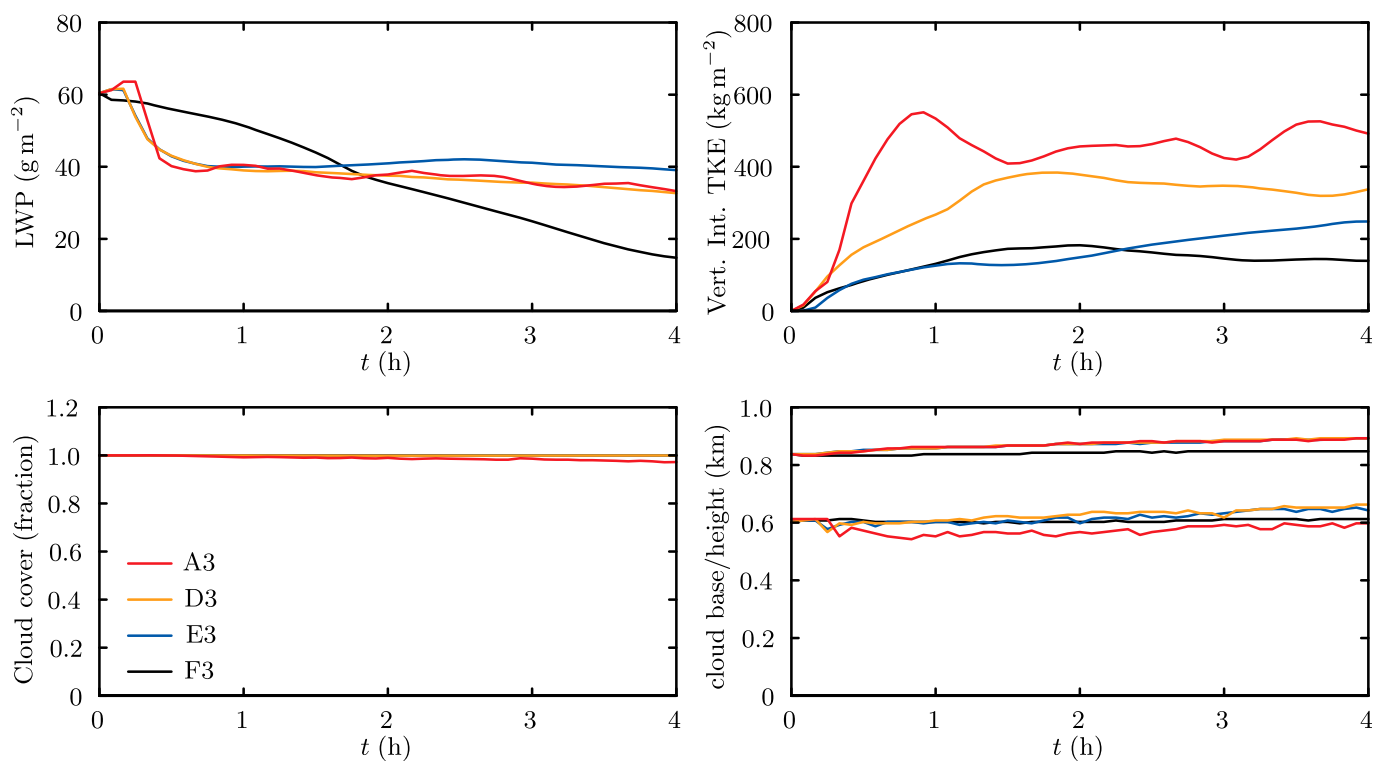

FIG. 8. Time traces of liquid water path, vertically integrated turbulent kinetic energy, cloud cover, and cloud-top and cloud-base height for cases A3, D3, E3, and F3. All cases have $\Delta x=5 \mathrm{~m}$. Case A3 corresponds to the base case, and cases D3, E3, and F3 have zero surface sensible and latent heat fluxes. Case E3 has no mean surface wind shear, whereas case F3 has no radiation, turbulence is only generated by surface shear. Note that cases D3, E3, and F3 have zero large-scale divergence, thus differences in entrainment rate between case A3 and D3-F3 cannot be readily inferred from the growth rate of the cloud-top height.

outside of the local bounds. In regions of strongly stratified flow, the SGS fluxes can become small or even zero, thus no dissipation is present leading to the creation numerical artifacts because excessively small scales are generated by the turbulence cascade. Figure 10 compares case H3, which uses nondissipative scalar advection, with the base case A3 of the same resolution. The numerical error affects the mean profiles as a $\theta_{l}$ overshoot and a $q_{t}$ undershoot with respect to the free-tropospheric profile above the inversion.

Because a relatively large numerical error is occurring in a critical part of the flow, it can affect the evolution of the boundary layer. The profile differences for cases A3 and H3 at $t=4 \mathrm{~h}$ (Fig. 10) show small differences. The most significant difference is the boundary layer growth rate between the two cases with the nondissipative centered scheme resulting in a $9.5-\mathrm{m}$-deeper boundary layer by $t=4 \mathrm{~h}$.

Typically scalars show higher sensitivity to finite difference scheme dispersion errors compared to the velocity field. Accordingly, no numerical artifacts are observed in the $u$ and $v$ profiles, even though a nondissipative scheme is used for momentum advection.

\section{e. Entrainment}

The entrainment rate is estimated using the kinematic estimate (e.g., Stevens 2002)

$$
E(t)=d h / d t+D h(t),
$$

where $h(t)$ is the height where $\left\langle q_{t}\right\rangle=5 \mathrm{~g} \mathrm{~kg}^{-1}$ and $D$ is the large-scale divergence, which for some cases is zero (see Table 2).

Figures 11 and 12 show the entrainment rates for the two grid resolution studies, the base (series A) and the cases without radiation (series B), respectively. Because of undulations of the inversion and the time differencing of $h$, the entrainment rate traces are not smooth. In spite of the jagged curves, Figs. 11 and 12 show finer differences between the LES runs compared to the cloud-top time traces of Figs. 2 and 4, in addition to quantifying $E(t)$. For runs without radiation, entrainment is essentially identical for all grid resolutions. For the base case the lowestresolution run, $\Delta x=20 \mathrm{~m}$, somewhat underentrains (in a time averaged sense, Fig. 2) with the remaining simulations being in good agreement. The entrainment rate for the series A runs is somewhat higher than the estimate based on the observations $4 \times 10^{-3} \mathrm{~m} \mathrm{~s}^{-1}$ (Stevens et al. 2003b).

The entrainment rate strongly depends on the presence of radiation in the LES and differs by about a factor of two between series A and B. However, it appears to weakly depend on the LWP in the range of the present simulations. The underentrainment of run A5 may be because LWP is too low $\left(\sim 10 \mathrm{~g} \mathrm{~m}^{-2}\right)$ to provide significant radiative forcing.

The grid resolution study of the entrainment rate does not support the conclusions of Bretherton et al. (1999) and Stevens and Bretherton (1999) that the fine structure 

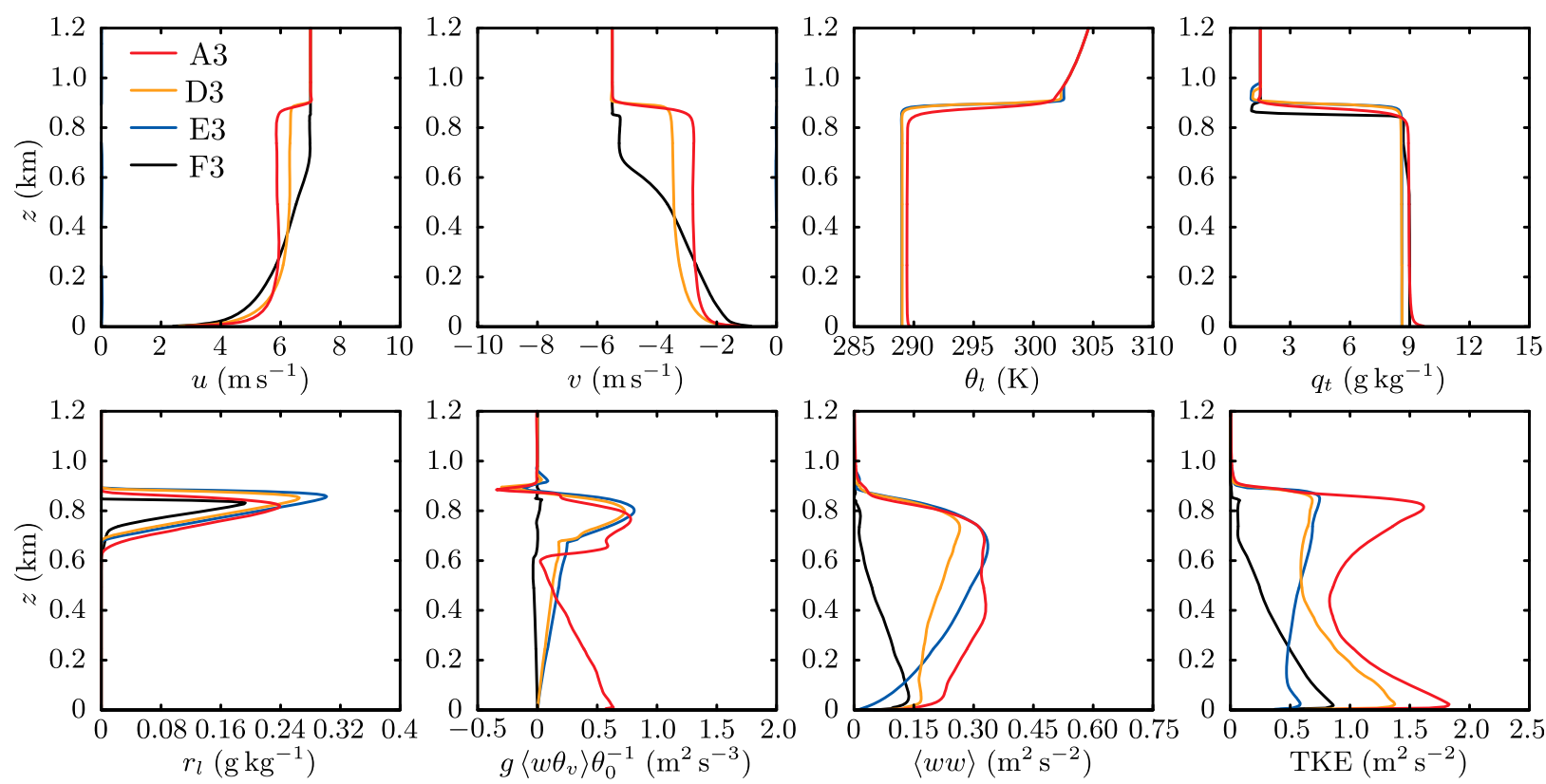

FIG. 9. Profiles of zonal wind $u$ and meridional wind $v$, liquid water potential temperature $\theta_{l}$, total water mixing ratio $q_{t}$, liquid water mixing ratio $r_{l}$, buoyancy flux $g\left\langle w \theta_{v}\right\rangle \theta_{0}^{-1}$, vertical velocity variance $\langle w w\rangle$, and turbulent kinetic energy (TKE) at $t=4 \mathrm{~h}$ (no time averaging) for cases A3, D3, E3, and F3. Turbulence statistics include the subgrid-scale contribution. The mean wind profiles for case E3 are zero.

of the inversion must be resolved $(\Delta z<10 \mathrm{~m})$. Thus, the sensitivity of the entrainment rate with respect to grid resolution for some LES models is specific to the turbulence parameterization and not a general property of the
LES methodology. In general, the presence of inertial range scaling at the grid scale is not a requirement of the LES methodology, as often stated (e.g., Mellado et al. 2018), but an assumption in specific parameterizations
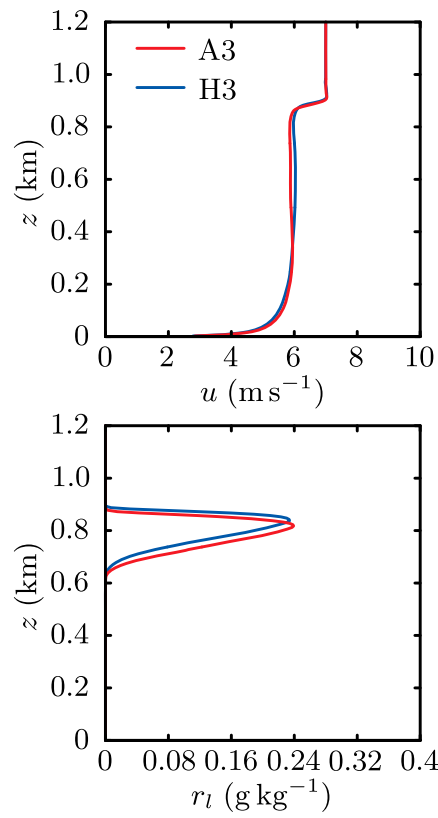
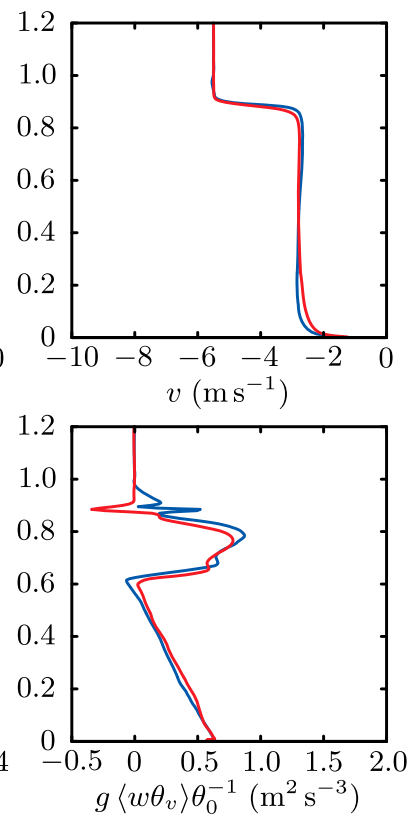
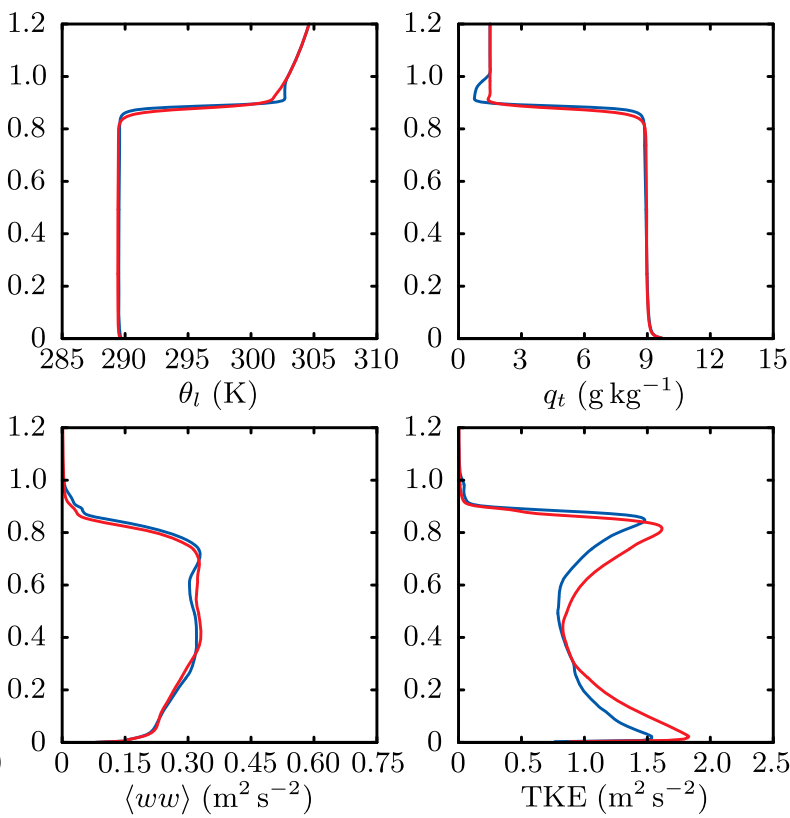

FIG. 10. Profiles of zonal wind $u$ and meridional wind $v$, liquid water potential temperature $\theta_{l}$, total water mixing ratio $q_{t}$, liquid water mixing ratio $r_{l}$, buoyancy flux $g\left\langle w \theta_{v}\right\rangle \theta_{0}^{-1}$, vertical velocity variance $\langle w w\rangle$, and turbulent kinetic energy (TKE) at $t=4 \mathrm{~h}$ (no time averaging) for cases A3 and $\mathrm{H} 3$. Turbulence statistics include the subgrid-scale contribution. The difference in cloud-top height between the two runs at $t=4 \mathrm{~h}$ is $9.5 \mathrm{~m}$. 


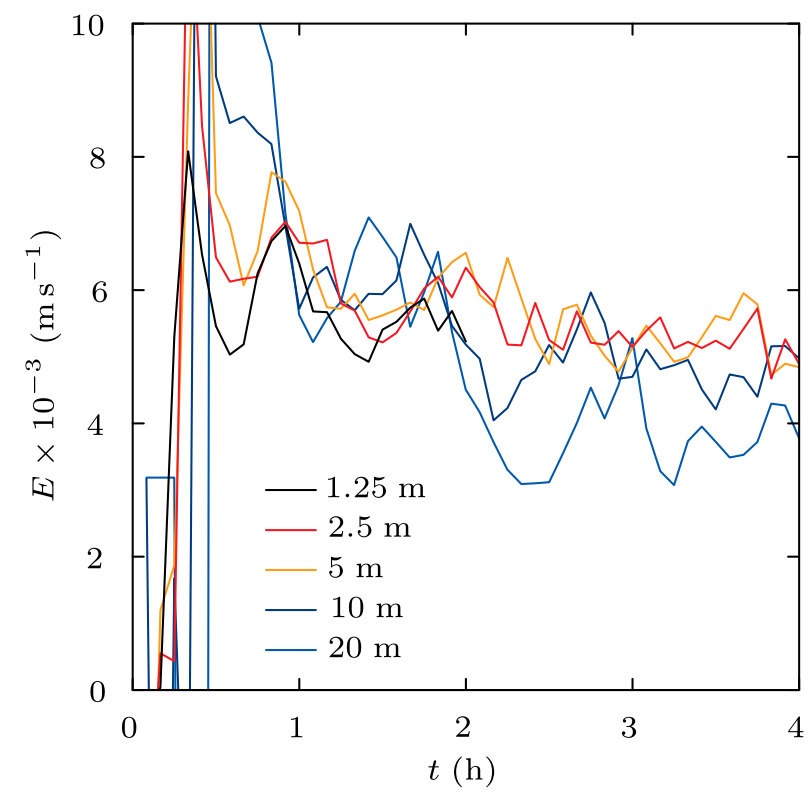

FIG. 11. Grid convergence of entrainment rate for series A runs.

(e.g., Lilly 1967). For instance, the buoyancy adjusted stretched vortex SGS model used in the current simulations does not assume inertial range scaling at the grid scale (Chung and Matheou 2014, their Fig. 4). Presently, LES yields grid-independent entrainment estimates without resolving the finescale structure, which conforms to one of the hallmarks of turbulence modeling: the largescale properties are the rate controlling parameters.

Figure 13 compares $E(t)$ for cases C3, D3, E3, F3, and H3. Most likely by coincidence, run C3 (modified buoyancy case) has similar $E(t)$ with runs D3 and H3. A comparison of run $\mathrm{C} 3$ and the corresponding run $\mathrm{A} 3$ (Fig. 11) shows that the modified buoyancy definition leads to a reduction of $E$ by about $40 \%$.

Runs D3 and E3 differ with respect to surface shear $\left(\mathbf{u}_{g}=0\right.$ in E3) but have statistically identical $E(t)$. The flow visualizations of Matheou et al. (2017) show that surface shear only affects the flow structure near the surface. Moreover, the TKE profiles for cases D3 and E3 (Fig. 9) differ only in the lower half of the boundary layer. Even though for the present case surface shear affects the flow structure, including TKE and $\langle w w\rangle$ (see Figs. 8 and 9), it has no effect on the entrainment rate.

Turbulence in case F3 is only generated by surface shear and for the duration of the run the entrainment rate is negligible. This does not imply zero entrainment for the shear-driven case because, as shown in Fig. 9, turbulence has not "diffused" to the boundary layer top by $t=4 \mathrm{~h}$. In all other cases, because of convective motions, sheargenerated turbulence is quickly transported to the boundary layer top and momentum becomes well mixed (Fig. 9).

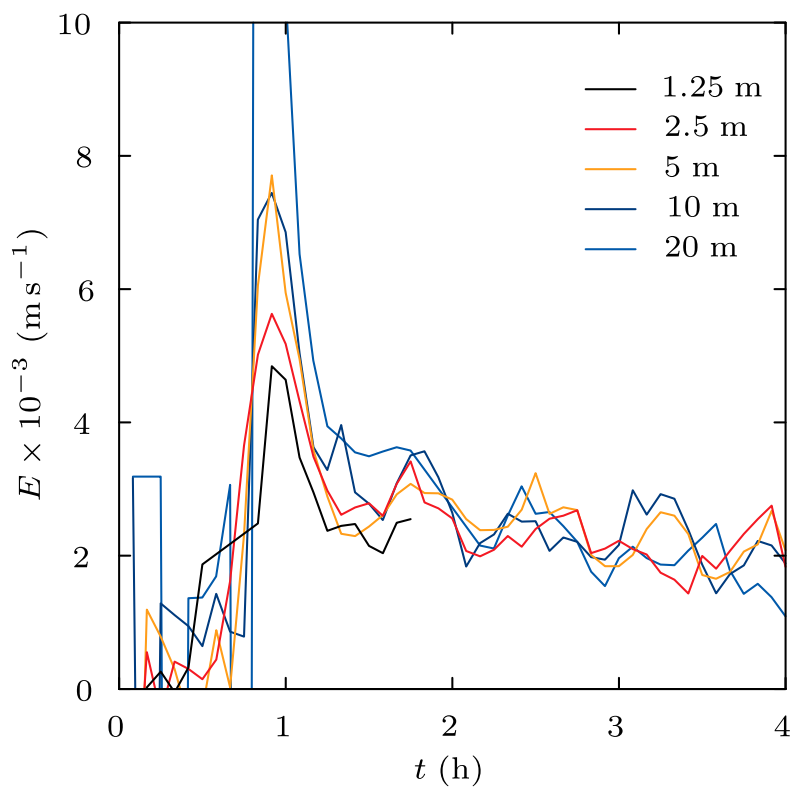

FIG. 12. Grid convergence of entrainment rate for series B runs.

Case $\mathrm{H}$, which uses a centered advection scalar advection discretization, has about $10 \%$ higher entrainment rate than the A series cases, with the difference being constant for the duration of the run following the initial model "spin up."

Overall, for the present cases the entrainment rate shows no dependence on LWP directly, except some sensitivity to LWP for very low values $<20 \mathrm{~g} \mathrm{~m}^{-2}$. However, strong $E(t)$ dependence is observed with

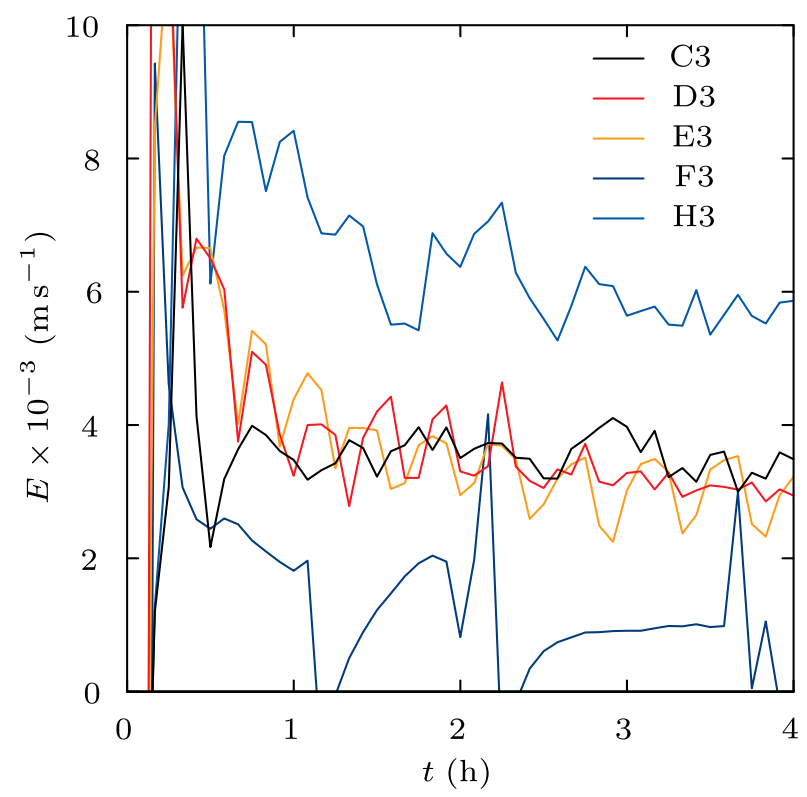

FIG. 13. Entrainment rates for C3, D3, E3, F3, and H3 runs. 

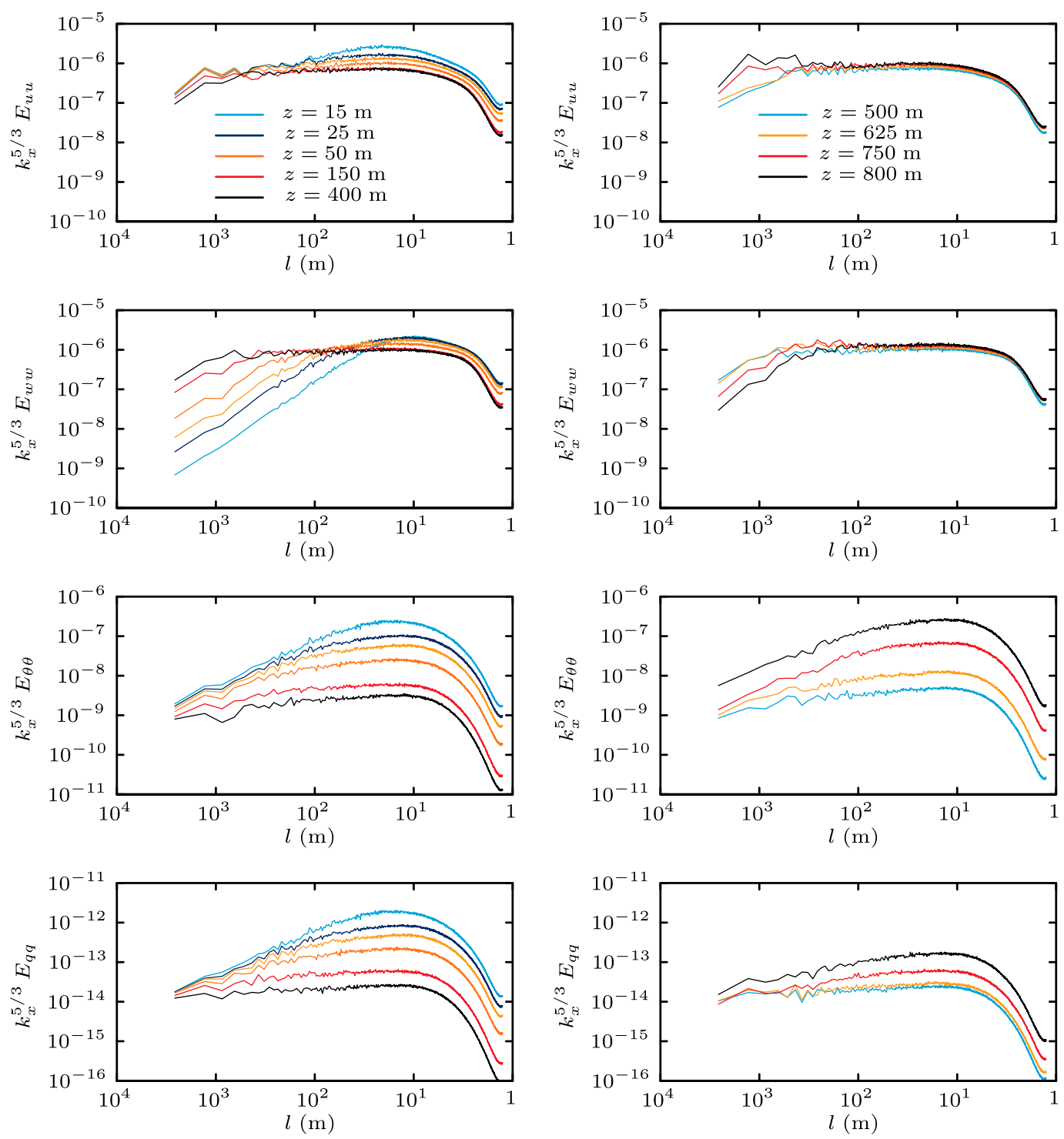

FIG. 14. Compensated spectra of (from top to bottom) zonal wind, vertical velocity, liquid water potential temperature, and total water mixing ratio taken along the zonal direction at various heights. (left) Spectra from the surface to about half-height of the boundary layer and (right) half-height to the top. All spectra are constructed from the instantaneous fields at $t=2 \mathrm{~h}$.

respect to processes that depend on the cloud liquid: radiation and buoyancy.

\section{f. Spectra}

The multiscale character of turbulence is examined through the spectra of $u, w, \theta_{l}$, and $q_{t}$. Data from run G1 at $t=2 \mathrm{~h}$ are used (no time averaging is performed). The one-dimensional energy spectra are computed by taking the two-dimensional Fourier transform of a variable $\phi$ $(x, y, z)$ at constant $z, \hat{\phi}\left(k_{x}, k_{y}, z\right)$, and then averaging across $k_{y}$ to form $E_{\phi \phi}\left(k_{x}, z\right)$, where $k_{x}$ is the wavenumber along direction $x$. This procedure reduces some of the aliasing of the $k_{y}$ modes onto the low $k_{x}$ wavenumbers, which is present when spectra are computed using one-dimensional Fourier transforms.

Figure 14 shows compensated spectra at different heights, thus horizontal parts of the spectra correspond to a $\sim k^{-5 / 3}$ scaling. The left-column panels show spectra at different heights in the lower half of the boundary layer and the right column in the upper half. The labels of the $x$ axis are converted to horizontal length scale, $l=\pi / k$, to assist the connection with the boundary layer physics. However, the $x$ axis remains in terms of increasing wavenumber, therefore the length scale decreases to the right. 
All spectra decrease quickly at small scales because of the implicit filtering property of the finite difference scheme (e.g., Matheou 2016). The beginning of the decreasing part of $E_{\theta \theta}$ and $E_{q q}$ occurs at larger length scales compared to $E_{u u}$ and $E_{w w}$ and the drop in spectral energy is higher because of the numerical dissipation introduced by the scalar advection scheme. The location of the departure from the inertial range scaling denotes the effective turbulence resolution, which is about $5 \Delta x$ for the velocity field and $8 \Delta x$ for scalars in the present simulations.

A power-law scaling of all variables is observed for spectra in the region of boundary layer half height. The scaling is $\sim k^{-5 / 3}$ for $u$ and $w$ (appearing as horizontal curves in the compensated spectra shown in Fig. 14). Plante et al. (2016) also observe $\sim k^{-5 / 3}$ scaling of velocity component spectra in the boundary layer in the analysis of the Physics of Stratocumulus Top (POST) research campaign observations. The scaling exponent is somewhat smaller in absolute value for $E_{\theta \theta}$ and $E_{q q}$. The large scales (low wavenumbers) of $E_{\theta \theta}$ and $E_{q q}$ and especially $E_{w w}$ are affected by the flow confinement near the surface and inversion. The effect is stronger for $w$ since the vertical velocity must approach zero near the surface and inversion. From the spectra can be inferred that the fluctuations of $\theta_{l}, q_{t}$, and $w$ decrease faster as the length scale increases near the surface compared to the region below the inversion, because at the surface $w=0$ and no large-scale variations of $q_{t}$ and $\theta_{l}$ are present when the sea surface temperature is uniform. In contrast, undulations of the inversion surface can cause large-scale $w$ variations and the combination of largescale convective motions and entrainment can result in large-scale fluctuations of $q_{t}$ and $\theta_{l}$ below the inversion (Matheou et al. 2017).

The low-wavenumber range of the $w$ spectra for $z<50 \mathrm{~m}$ has zero slope (i.e., $k^{5 / 3}$ slope is observed for the compensated spectra in Fig. 14). In the same region, $E_{\theta \theta}$ and $E_{q q}$ have small in absolute value negative slopes that approach $\sim k^{-5 / 3}$ for increasing $z$.

Spectra in previous LES studies (Stevens et al. 2000; Pedersen et al. 2016, 2018) do not exhibit a well-defined power-law scaling, likely because of the limited number of grid points and coarse resolution used. Liquid water mixing ratio spectra for case G1 are discussed in Matheou (2018).

Overall, in spite of the turbulence being anisotropic and inhomogeneous in the vertical direction, the spectra show robust inertial range scaling for at least two decades of length scales.

\section{Conclusions}

The effects of physical processes and numerical model parameters on large-eddy simulations of the nocturnal stratocumulus observed during DYCOMS-II RF01 (Stevens et al. 2003a, 2005) are investigated using a series of "perturbation" numerical experiments where both physical and numerical model parameters are varied. Overall, the simulations show a delicate balance with physical-process sensitivities amplified by numerical model features. The delicate nature of the simulations is likely a consequence of the design of the case, which is susceptible to decoupling (Stevens et al. 2003a).

A grid converged simulation that agrees with the observations of Stevens et al. (2005) is only possible at very fine grid resolutions $\Delta x<2.5 \mathrm{~m}$. However, the simulations also show some robust features, such as the grid resolution independence of entrainment rate and mean profiles (except cloud liquid).

In spite of the fine grid resolution requirement for the convergence of the cloud liquid, the present results are encouraging for the LES methodology. For the first time, grid convergence and agreement with all observations in Stevens et al. (2005), especially with respect to cloud liquid and vertical velocity variance [comparison with $\langle w w w\rangle$ is shown in Matheou (2018)] is achieved without any model tuning or ad hoc model choices. The model is used in a standard configuration identical to the simulations in Matheou and Chung (2014) and does not include any flow adjustable parameters. The grid-converged numerical solution illustrates that reliable and accurate modeling of Sc is attainable.

The study is structured around three main questions posed in the Introduction. The main deductions can be summarized as follows.

Why is grid convergence difficult to achieve? The present simulations show a strong feedback between cloud liquid amount, cloud-top radiative cooling, and turbulence in the boundary layer. Because cloud liquid $r_{l}$ is diagnosed from the two conserved variables, the total water mixing ratio $q_{t}$ and liquid water potential temperature $\theta_{l}$, even relatively small $q_{t}$ and $\theta_{l}$ errors can result in large $r_{l}$ error. In turn, the radiative flux depends exponentially on $r_{l}$, thus small errors are amplified resulting in large buoyancy forcing near the boundary layer top. In contrast, when the $r_{l}$-radiationbuoyancy feedback is not present (i.e., in simulations without radiation) the turbulence structure of the boundary layer remains essentially identical for grid resolutions $\Delta x=1.25-20 \mathrm{~m}$, even though the amount of cloud liquid varies significantly with $\Delta x$. Grid convergence properties depend on the model formulation, thus other LES models may exhibit different convergence characteristics.

What parameters control the cloud cover and boundary layer turbulence in the LES? In the base case, cloud cover, 

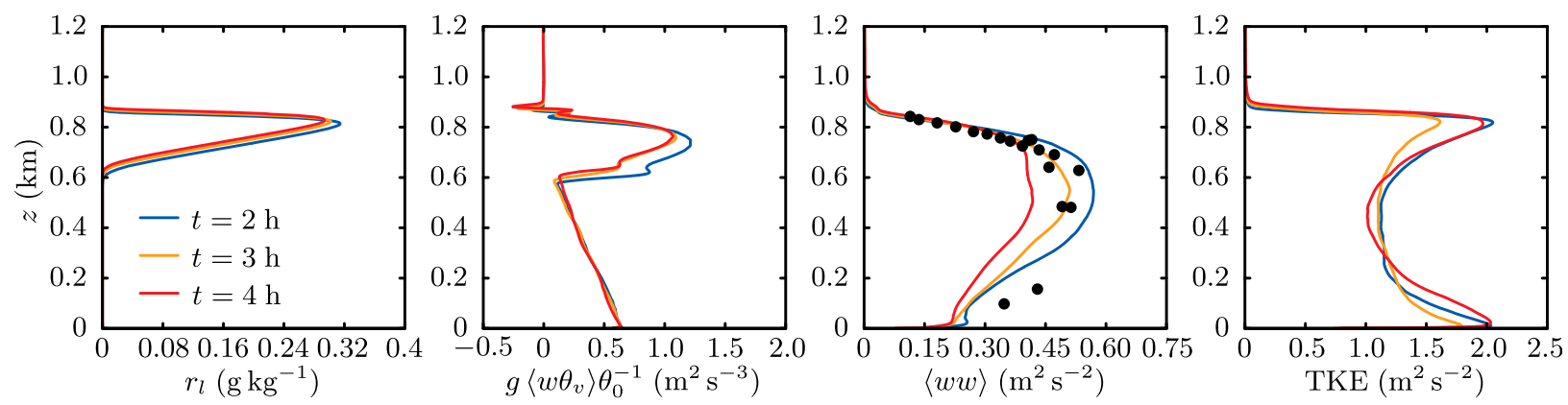

FIG. B1. Profiles of liquid water mixing ratio $r_{l}$, buoyancy flux $g\left\langle w \theta_{v}\right\rangle \theta_{0}^{-1}$, vertical velocity variance $\langle w w\rangle$, and turbulent kinetic energy (TKE) at $t=2,3$, and $4 \mathrm{~h}$ (no time averaging) for case A2. Circles denote observations.

liquid water path, and turbulent fluxes primarily depend on grid resolution because of the cloud liquid-radiationbuoyancy feedback. Even though the initial condition is unstable with respect to the Cloud Top Entrainment Instability (CTEI) criterion (Deardorff 1980; Randall 1980; Stevens et al. 2003a), all sufficiently resolved simulations maintain a solid cloud deck for the duration of the run, including cases with zero surface sensible and latent heat fluxes. This is consistent with cloud-top process-scale modeling results which suggest that cloud-top evaporative cooling cannot lead to a runaway cloud evaporation (Mellado 2010; Van der Dussen et al. 2014). Even though cloud-top radiative cooling is presented as a central attribute of stratocumulus (e.g., Wood 2012; Mellado 2017), the present simulations suggest all three main sources of turbulence (surface buoyancy flux, cloud-top radiative cooling, and surface shear) substantially contribute to the total vertically integrated turbulent kinetic energy (TKE) of the boundary layer. For the duration of the runs, simulations without radiative cooling maintain a stratiform cloud and well-mixed mean profiles but with a decoupled buoyancy flux profile. The present study shows unequivocally (likely for the first time, since it uniquely blends numerical convergence and convergence toward observations) the essential role that cloud-top radiative cooling plays in the turbulent structure of the stratocumulus boundary layer.

How does entrainment depend on numerical model and physical choices in the LES? In contrast to previous stratocumulus simulations (Bretherton et al. 1999; Stevens and Bretherton 1999; Stevens et al. 2000) the entrainment rate does not depend on grid resolution, similar to LES of "dry" (without condensate) convective boundary layers (e.g., Sullivan and Patton 2011; Matheou and Chung 2014). In agreement with the model ensemble results of Stevens et al. (2005), the entrainment rate does not depend on LWP when LWP $>20 \mathrm{~g} \mathrm{~m}^{-2}$, but the degree of decoupling (based on the minimum of the buoyancy flux near cloud base) continuously depends on LWP. Entrainment was observed to strongly depend on the physical processes (e.g., radiation, surface buoyancy flux, and buoyancy formulation). The buoyancy perturbation run suggests that the buoyancy reversal instability of the cloud top significantly enhances the entrainment rate. Entrainment was observed to somewhat depend on the properties of the scalar advection scheme because of numerical artifacts near the inversion. Overall, the present results challenge some of the conclusions of previous studies that the inversion structure details must be explicitly resolved.

Turbulence spectra of the velocity components, liquid water potential temperature, and total water mixing ratio exhibit inertial range scaling away from the confinement effects of the surface and inversion. The scaling exponent, which spans more than two decades, is $-5 / 3$ for the velocity components and somewhat smaller in absolute value for the total water mixing ratio and the liquid water potential temperature.

Acknowledgments. We acknowledge fruitful discussions with Prof. C. Bretherton (University of Washington). We acknowledge the support provided by the Office of Naval Research, Marine Meteorology Program, the NASA MAP Program, the NOAA/CPO MAPP Program, and by the DOE Office of Biological and Environmental Research, Earth and Environmental System Modeling Program. Computational resources supporting this work were provided by the NASA High-End Computing (HEC) Program through the NASA Advanced Supercomputing (NAS) Division at Ames Research Center. The research presented in this paper was supported by the systems, services, and capabilities provided by the University of Connecticut High Performance Computing (HPC) facility. Part of this research was carried out at the Jet Propulsion Laboratory, California Institute of Technology, under a contract with the National Aeronautics and Space Administration. 


\section{APPENDIX A}

\section{Numerical Discretization}

The governing equations [Eqs. (1)-(4)] are discretized on an Arakawa C (staggered) grid (Harlow and Welch 1965; Arakawa and Lamb 1977; Matheou et al. 2011). The fully conservative four-order advection scheme of Morinishi et al. (1998) is used for momentum advection, the Quadratic Upstream Interpolation for Convective Kinematics (QUICK; Leonard 1979) is used for $\theta_{l}$ and $q_{t}$ advection for all simulations except case $\mathrm{H} 3$, which utilizes the centered-differences fourth-order advection of Morinishi et al. (1998). For all cases, regardless of the order of the advection scheme, second-order centered differences are used to approximate the spatial derivatives of the subgrid-scale model terms. The semidiscrete system of equations is advanced in time using the thirdorder Runge-Kutta of Spalart et al. (1991).

QUICK (Leonard 1979), a linear upwinding scheme, is used for scalar advection. QUICK does not enforce monotonicity (positive definiteness) of the advected scalar fields and it is less dissipative than monotone schemes (e.g., Matheou and Dimotakis 2016). Thus, QUICK is presently viewed as a compromise between nondissipative schemes, which produce large numerical errors and positive definite schemes that introduce large quantities of numerical dissipation.

The scalar advection terms are approximated by

$$
\frac{\partial u \phi}{\partial x} \approx \frac{9}{8} \frac{f_{i+1 / 2}-f_{i-1 / 2}}{\Delta x}-\frac{1}{8} \frac{g_{i+3 / 2}-g_{i-3 / 2}}{3 \Delta x},
$$

where

$$
\begin{aligned}
& f_{i+1 / 2}=u_{i+1 / 2}\left[\left(\phi_{i}+\phi_{i+1}\right) / 2+\phi_{u}\right], \\
& g_{i+1 / 2}=u_{i+1 / 2}\left(\phi_{i-1}+\phi_{i+2}\right) / 2 .
\end{aligned}
$$

The upwind contribution to the scalar flux is

$$
\phi_{u}= \begin{cases}\left(\phi_{i-1}-2 \phi_{i}+\phi_{i+1}\right) / 8, & \text { if } u_{i+1 / 2}>0 \\ \left(\phi_{i}-2 \phi_{i+1}+\phi_{i+2}\right) / 8, & \text { if } u_{i+1 / 2}<0 .\end{cases}
$$

\section{APPENDIX B}

\section{Time Variability of Boundary Layer Profiles}

The highest-resolution cases A1 and B1 are only ran up to $t=2 \mathrm{~h}$ instead of the full length of the simulation to $t=4 \mathrm{~h}$ as the other cases. To assess the
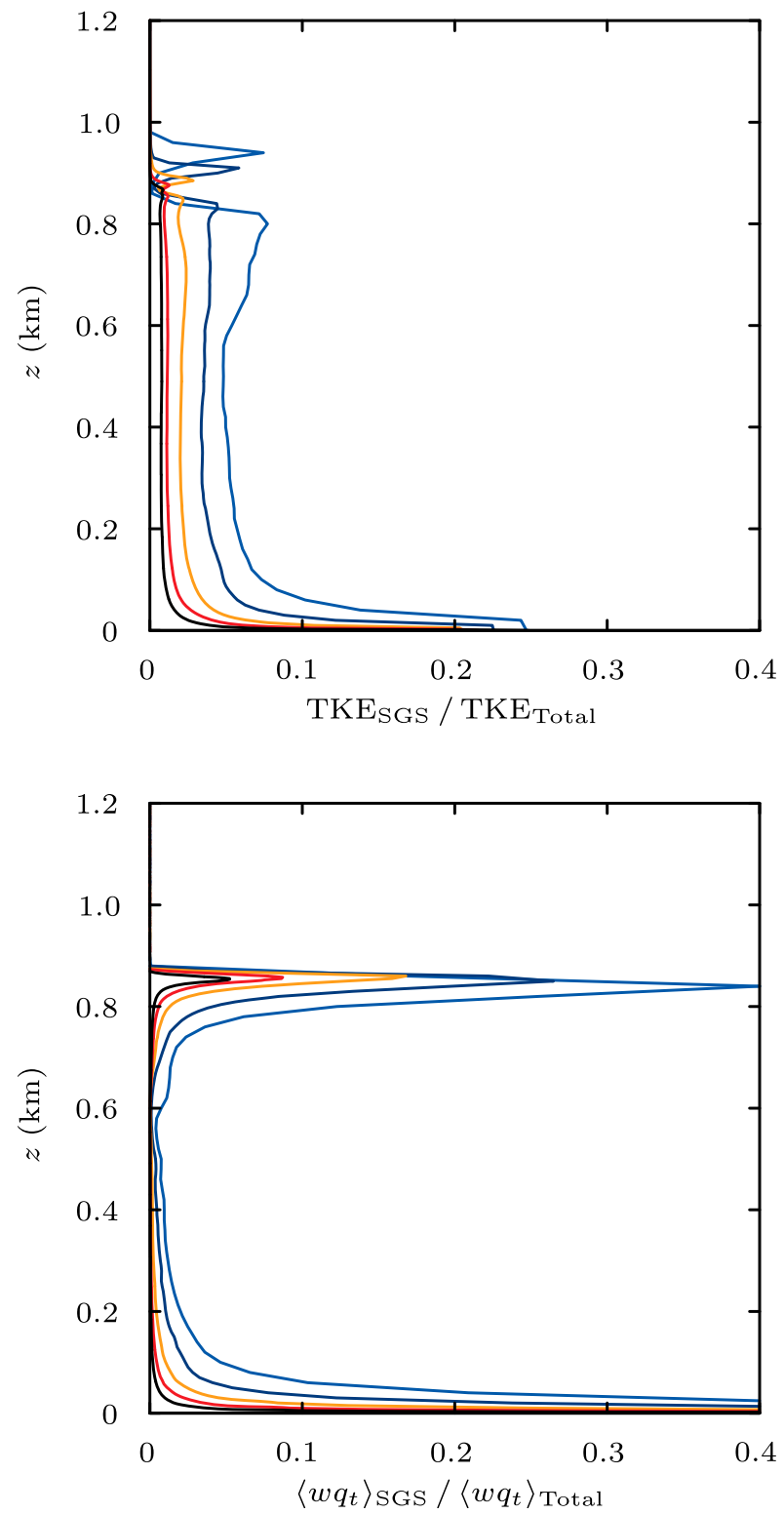

FIG. C1. Subgrid-scale fraction of turbulent kinetic energy and total water vertical flux at $t=2 \mathrm{~h}$ (no time averaging) for series A runs. Lines are as in Fig. 3.

representativeness of flow statistics at $t=2 \mathrm{~h}$, Fig. B1 compares profiles of four second-order statistics for case A2 (the highest-resolution case ran the full length) at $t=2,3$, and $4 \mathrm{~h}$. Even though the boundary layer is not statistically stationary and slowly evolves for the entire duration of the simulation, the differences between $t=2 \mathrm{~h}$ and $t=4 \mathrm{~h}$ are small. The largest differences are observed in the vertical velocity variance, which decreases with time. The profiles at $t=3 \mathrm{~h}$, the intermediate value of the $\langle w w\rangle$ profiles, are in better agreement with the observational data. Thus, agreement $\langle w w\rangle$ 
between LES and observations in Fig. 3 likely improves for later times.

\section{APPENDIX C}

\section{Subgrid-Scale Turbulent Kinetic Energy and Moisture Flux Fractions}

The fraction of the subgrid-scale (SGS) turbulent kinetic energy and total water vertical flux for case A runs at $t=2 \mathrm{~h}$ (no time averaging) are shown in Fig. C1. For all grid resolutions the SGS contributions are small away from the inversion and surface. Turbulence becomes less resolved with respect to $\Delta x$ near the inversion and surface because the vertical scale of the largest turbulent motions is reduced. The three lowest-resolution runs exhibit spurious TKE spikes above the inversion. The total water turbulent flux SGS fractions follow the grid resolution criterion of Matheou and Chung (2014): about $90 \%$ of the turbulent flux must be resolved in stably stratified turbulent flow LES.

\section{REFERENCES}

Arakawa, A., and V. R. Lamb, 1977: Computational design of the basic dynamical processes of the UCLA general circulation model. Methods of Computational Physics, J. Chang, Ed., Vol. 17, Academic Press, 173-265.

Blossey, P. N., and Coauthors, 2013: Marine low cloud sensitivity to an idealized climate change: The CGILS LES intercomparison. J. Adv. Model. Earth Syst., 5, 234-258, https://doi.org/10.1002/ jame.20025.

Bony, S., and J.-L. Dufresne, 2005: Marine boundary layer clouds at the heart of tropical cloud feedback uncertainties in climate models. Geophys. Res. Lett., 32, L20806, https:// doi.org/10.1029/2005GL023851.

Bretherton, C. S., 1997: Convection in stratocumulus-topped atmospheric boundary layers. The Physics and Parameterization of Moist Atmospheric Convection, R. K. Smith, Ed., Springer, $127-142$.

_ 2015: Insights into low-latitude cloud feedbacks from highresolution models. Philos. Trans. Roy. Soc. London A, 373, 20140415, https://doi.org/10.1098/rsta.2014.0415.

__, and Coauthors, 1999: An intercomparison of radiatively driven entrainment and turbulence in a smoke cloud, as simulated by different numerical models. Quart. J. Roy. Meteor. Soc., 125, 391-423, https://doi.org/10.1002/qj.49712555402.

Charnock, H., 1955: Wind stress over a water surface. Quart. J. Roy. Meteor. Soc., 81, 639-640, https://doi.org/10.1002/ qj.49708135027.

Chung, D., and G. Matheou, 2014: Large-eddy simulation of stratified turbulence. Part I: A vortex-based subgrid-scale model. J. Atmos. Sci., 71, 1863-1879, https://doi.org/10.1175/ JAS-D-13-0126.1.

_,- , and J. Teixeira, 2012: Steady-state large-eddy simulations to study the stratocumulus to shallow-cumulus cloud transition. J. Atmos. Sci., 69, 3264-3276, https://doi.org/10.1175/ JAS-D-11-0256.1.
Cuijpers, J. W. M., and P. G. Duynkerke, 1993: Large-eddy simulation of trade-wind cumulus clouds. J. Atmos. Sci., 50 , 3894-3908, https://doi.org/10.1175/1520-0469(1993)050<3894: LESOTW $>2.0 . \mathrm{CO} ; 2$.

de Lozar, A., and J. P. Mellado, 2015: Mixing driven by radiative and evaporative cooling at the stratocumulus top. J. Atmos. Sci., 72, 4681-4700, https://doi.org/10.1175/JAS-D-15-0087.1.

Deardorff, J. W., 1980: Cloud top entrainment instability. J. Atmos. Sci., 37, 131-147, https://doi.org/10.1175/1520-0469(1980) 037<0131:CTEI>2.0.CO;2.

Harlow, F. H., and J. E. Welch, 1965: Numerical calculation of timedependent viscous incompressible flow of fluid with free surface. Phys. Fluids, 8, 2182-2189, https://doi.org/10.1063/1.1761178.

Hartmann, D. L., M. E. Ockert-Bell, and M. L. Michelsen, 1992: The effect of cloud type on Earth's energy balance: Global analysis. J. Climate, 5, 1281-1304, https://doi.org/10.1175/15200442(1992)005<1281:TEOCTO > 2.0.CO;2.

Heinze, R., D. Mironov, and S. Raasch, 2015: Second-moment budgets in cloud topped boundary layers: A large-eddy simulation study. J. Adv. Model. Earth Syst., 7, 510-536, https:// doi.org/10.1002/2014MS000376.

Lenschow, D. H., and Coauthors, 1988: Dynamics and chemistry of marine stratocumulus (DYCOMS) experiment. Bull. Amer. Meteor. Soc., 69, 1058-1067, https://doi.org/10.1175/1520-0477(1988) 069<1058:DACOMS > 2.0.CO;2.

Leonard, B. P., 1979: A stable and accurate convective modelling procedure based on quadratic upstream interpolation. Comput. Methods Appl. Mech. Eng., 19, 59-98, https://doi.org/ 10.1016/0045-7825(79)90034-3.

Lilly, D. K., 1967: The representation of small-scale turbulence in numerical simulation experiments. Proc. IBM Scientific Computing Symp. on Environmental Sciences, Yorktown Heights, NY, IBM, 195-210.

Malinowski, S. P., and Coauthors, 2013: Physics of Stratocumulus Top (POST): Turbulent mixing across capping inversion. Atmos. Chem. Phys., 13, 12 171-12 186, https://doi.org/10.5194/ acp-13-12171-2013.

Matheou, G., 2016: Numerical discretization and subgrid-scale model effects on large-eddy simulations of a stable boundary layer. Quart. J. Roy. Meteor. Soc., 142, 3050-3062, https:// doi.org/10.1002/qj.2888.

, 2018: Turbulence structure in a stratocumulus cloud. Atmosphere, 9, 392, https://doi.org/10.3390/atmos9100392.

—, and D. Chung, 2014: Large-eddy simulation of stratified turbulence. Part II: Application of the stretched-vortex model to the atmospheric boundary layer. J. Atmos. Sci., 71, 4439 4460, https://doi.org/10.1175/JAS-D-13-0306.1.

— simulations. J. Comput. Phys., 327, 97-120, https://doi.org/ 10.1016/j.jcp.2016.08.035.

— D. Chung, and J. Teixeira, 2017: Large-eddy simulation of a stratocumulus cloud. Phys. Rev. Fluids, 2, 090509, https:// doi.org/10.1103/PhysRevFluids.2.090509.

,$- \ldots$, L. Nuijens, B. Stevens, and J. Teixeira, 2011: On the fidelity of large-eddy simulation of shallow precipitating cumulus convection. Mon. Wea. Rev., 139, 2918-2939, https:// doi.org/10.1175/2011MWR3599.1.

Mellado, J. P., 2010: The evaporatively driven cloud-top mixing layer. J. Fluid Mech., 660, 5-36, https://doi.org/10.1017/ S0022112010002831.

, 2017: Cloud-top entrainment in stratocumulus clouds. Annu. Rev. Fluid Mech., 49, 145-169, https://doi.org/10.1146/ annurev-fluid-010816-060231. 
— C. S. Bretherton, B. Stevens, and M. C. Wyant, 2018: DNS and LES for simulating stratocumulus: Better together. J. Adv. Model. Earth Syst., 10, 1421-1438, https://doi.org/ 10.1029/2018MS001312.

Moeng, C.-H., 2000: Entrainment rate, cloud fraction, and liquid water path of PBL stratocumulus clouds. J. Atmos. Sci., 57, 3627-3643, https://doi.org/10.1175/1520-0469(2000)057<3627: ERCFAL $>2.0 . \mathrm{CO} ; 2$

_ S. Shen, and D. A. Randall, 1992: Physical processes within the nocturnal stratus-topped boundary layer. J. Atmos. Sci., 49, 2384-2401 https://doi.org/10.1175/1520-0469(1992)049<2384: PPWTNS $>2.0 . \mathrm{CO} ; 2$.

— , and Coauthors, 1996: Simulation of a stratocumulus-topped planetary boundary layer: Intercomparison among different numerical codes. Bull. Amer. Meteor. Soc., 77, 261-278, https:// doi.org/10.1175/1520-0477(1996)077<0261:SOASTP>2.0.CO;2.

Morinishi, Y., T. S. Lund, O. V. Vasilyev, and P. Moin, 1998: Fully conservative higher order finite difference schemes for incompressible flow. J. Comput. Phys., 143, 90-124, https:// doi.org/10.1006/jcph.1998.5962.

Ogura, Y., and N. A. Phillips, 1962: Scale analysis of deep and shallow convection in the atmosphere. J. Atmos. Sci., 19, 173-179, https://doi.org/10.1175/1520-0469(1962)019<0173: SAODAS $>2.0 . C O ; 2$.

Pedersen, J. G., S. P. Malinowski, and W. W. Grabowski, 2016: Resolution and domain-size sensitivity in implicit large-eddy simulation of the stratocumulus-topped boundary layer. J. Adv. Model. Earth Syst., 8, 885-903, https://doi.org/10.1002/ 2015MS000572.

_ - Y.-F. Ma, W. W. Grabowski, and S. P. Malinowski, 2018: Anisotropy of observed and simulated turbulence in marine stratocumulus. J. Adv. Model. Earth Syst., 10, 500-515, https:// doi.org/10.1002/2017MS001140.

Plante, I. J.-L., and Coauthors, 2016: Physics of Stratocumulus Top (POST): Turbulence characteristics. Atmos. Chem. Phys., 16, 9711-9725, https://doi.org/10.5194/acp-16-9711-2016.

Pressel, K. G., S. Mishra, T. Schneider, C. M. Kaul, and Z. Tan, 2017: Numerics and subgrid-scale modeling in large eddy simulations of stratocumulus clouds. J. Adv. Model. Earth Syst., 9, 1342-1365, https://doi.org/10.1002/2016MS000778.

Randall, D. A., 1980: Conditional instability of the first kind upside-down. J. Atmos. Sci., 37, 125-130, https://doi.org/10.1175/ 1520-0469(1980)037<0125:CIOTFK >2.0.CO;2.

Schmidt, H., and U. Schumann, 1989: Coherent structure of the convective boundary layer derived from large-eddy simulations. J. Fluid Mech., 200, 511-562, https://doi.org/10.1017/ S0022112089000753.

Siebesma, A. P., and Coauthors, 2003: A large eddy simulation intercomparison study of shallow cumulus convection. J. Atmos. Sci., 60, 1201-1219, https://doi.org/10.1175/1520-0469(2003)60<1201: ALESIS $>2.0 . \mathrm{CO} ; 2$.

Smolarkiewicz, P. K., and L. G. Margolin, 1998: MPDATA: A finite-difference solver for geophysical flows. J. Comput. Phys., 140, 459-480, https://doi.org/10.1006/jcph.1998.5901.
Spalart, P. R., R. D. Moser, and M. M. Rogers, 1991: Spectral methods for the Navier-Stokes equations with one infinite and two periodic directions. J. Comput. Phys., 96, 297-324, https:// doi.org/10.1016/0021-9991(91)90238-G.

Stevens, B., 2002: Entrainment in stratocumulus-topped mixed layers. Quart. J. Roy. Meteor. Soc., 128, 2663-2690, https:// doi.org/10.1256/qj.01.202.

, 2005: Atmospheric moist convection. Annu. Rev. Earth Planet. Sci., 33, 605-643, https://doi.org/10.1146/annurev.earth.33.092203.122658. and Coauthors, 2003a: Dynamics and chemistry of marine stratocumulus-DYCOMS-II. Bull. Amer. Meteor. Soc., 84, 579-593, https://doi.org/10.1175/BAMS-84-5-Stevens.

— , and Coauthors, 2003b: On entrainment rates in nocturnal marine stratocumulus. Quart. J. Roy. Meteor. Soc., 129, 34693493, https://doi.org/10.1256/qj.02.202.

_ , and Coauthors, 2005: Evaluation of large-eddy simulations via observations of nocturnal marine stratocumulus. Mon. Wea. Rev., 133, 1443-1462, https://doi.org/10.1175/MWR2930.1.

Stevens, D. E., and C. S. Bretherton, 1999: Effects of resolution on the simulation of stratocumulus entrainment. Quart. J. Roy. Meteor. Soc., 125, 425-439, https://doi.org/10.1002/ qj.49712555403.

- J. B. Bell, A. S. Almgren, V. E. Beckner, and C. A. Rendleman 2000: Small-scale processes and entrainment in a stratocumulus marine boundary layer. J. Atmos. Sci., 57, 567-581, https://doi.org/10.1175/1520-0469(2000)057<0567: SSPAEI $>2.0 . \mathrm{CO} ; 2$.

Sullivan, P. G., and E. G. Patton, 2011: The effect of mesh resolution on convective boundary layer statistics and structures generated by large-eddy simulation. J. Atmos. Sci., 68, 23952415, https://doi.org/10.1175/JAS-D-10-05010.1.

Sullivan, P. P., C.-H. Moeng, B. Stevens, D. H. Lenschow, and S. D. Mayor, 1998: Structure of the entrainment zone capping the convective atmospheric boundary layer. J. Atmos. Sci., 55, 3042-3064, https://doi.org/10.1175/1520-0469(1998)055<3042: SOTEZC $>2.0 . \mathrm{CO} ; 2$

Tsushima, Y., and Coauthors, 2016: Robustness, uncertainties, and emergent constraints in the radiative responses of stratocumulus cloud regimes to future warming. Climate Dyn., 46, 3025-3039, https://doi.org/10.1007/s00382-015-2750-7.

Van der Dussen, J. J., S. R. De Roode, and A. P. Siebesma, 2014: Factors controlling rapid stratocumulus cloud thinning. J. Atmos. Sci., 71, 655-664, https://doi.org/10.1175/JAS-D-130114.1.

Wood, R., 2012: Stratocumulus clouds. Mon. Wea. Rev., 140, 2373 2423, https://doi.org/10.1175/MWR-D-11-00121.1.

Yamaguchi, T., and D. A. Randall, 2008: Large-eddy simulation of evaporatively driven entrainment in cloud-topped mixed layers. J. Atmos. Sci., 65, 1481-1504, https://doi.org/10.1175/ 2007JAS2438.1.

, W. A. Brewer, and G. Feingold, 2013: Evaluation of modeled stratocumulus-capped boundary layer turbulence with shipborne data. J. Atmos. Sci., 70, 3895-3919, https://doi.org/ 10.1175/JAS-D-13-050.1. 